\title{
A theoretical unifying scheme for gamma-ray bright blazars
}

\author{
G. Ghisellini ${ }^{1}$, A. Celotti ${ }^{2,3}$ G. Fossati ${ }^{2}$, L. Maraschi $^{1}$ and A. Comastri ${ }^{4}$ \\ 1 Osservatorio Astronomico di Brera, via Bianchi 46, I-22055 Merate, Italy \\ 2 S.I.S.S.A., via Beirut 2-4, 34014 Trieste, Italy \\ 3 Institute of Astronomy, Madingley Road, Cambridge CBз оНА \\ ${ }^{4}$ Osservatorio Astronomico di Bologna, via Zamboni 33, I-40126, Bologna, Italy
}

Received $* * * ;$ in original form $* * *$

\begin{abstract}
The phenomenology of $\gamma$-ray bright blazars can be accounted for by a sequence in the source power and intensity of the diffuse radiation field surrounding the relativistic jet. Correspondingly, the equilibrium particle distribution peaks at different energies. This leads to a trend in the observed properties: an increase of the observed power corresponds to: 1) a decrease in the frequencies of the synchrotron and inverse Compton peaks; 2) an increase in the ratio of the powers of the high and low energy spectral components. Objects along this sequence would be observationally classified respectively as high frequency BL Lac objects, low frequency BL Lac objects, highly polarized quasars and lowly polarized quasars.

The proposed scheme is based on the correlations among the physical parameters derived in the present paper by applying to $51 \gamma$-ray loud blazars two of the most accepted scenarios for the broad band emission of blazars, namely the synchrotron self-Compton and external Compton models, and explains the observational trends presented by Fossati et al. (1998) in a companion paper, dealing with the spectral energy distributions of all blazars. This gives us confidence that our scheme applies to all blazars as a class.
\end{abstract}

Key words: galaxies: active - quasars - BL Lacertae objects - jets - gamma-rays: theory - radiation mechanisms: non-thermal

\section{INTRODUCTION}

Among Active Galactic Nuclei (AGN) blazars represent the most extreme and powerful sources. The fundamental property characterizing blazars is their beamed continuum, due to plasma moving relativistically along the line of sight.

This scenario seems to apply to objects with somewhat different observational properties leading to different classifications/definitions. Objects with significant emission line equivalent widths are usually found as flat spectrum radio quasars (FSRQ). Objects without emission lines $(\mathrm{EW}<5$ $\AA)$ are classified as BL Lac objects. Different flavors of BL Lac objects have been found in radio and X-ray surveys. These also correspond to differences in the overall spectral energy distributions (SED) (see. e.g. Padovani \& Giommi 1995), which have been interpreted either as due to orientation (Ghisellini \& Maraschi 1989, Urry \& Padovani 1995), or as intrinsic (Padovani \& Giommi 1995). Nevertheless, while different sub-classes have different average properties, the actual distinction among them is certainly fuzzy and so far

\footnotetext{
* E-mail: gabriele@merate.mi.astro.it
}

several sources have shown intermediate behavior. In fact arguments for a substantial 'continuity' in the continuum spectral properties leading to adopt the blazar denomination as including both, BL Lacs as well as FSRQs, have been recently re-proposed by Maraschi et al. (1995), Sambruna et al. (1996) and Fossati et al. (1997).

The recent discoveries of about $\sim 60$ blazars emitting in the $\gamma$-ray band, by EGRET on board the Compton GammaRay Observatory (CGRO) (Fichtel et al. 1994; von Montigny et al. 1995; Thompson et al. 1995; Mattox et al. 1997) and of a few BL Lac objects by WHIPPLE and HEGRA (Weekes et al. 1996, Petry et al. 1996), have revealed that the bulk of their radiative output is emitted in the $\gamma$-ray range, thus allowing us to discuss for the first time the characteristics of blazars knowing their total emission output and their entire SED. At the same time these observations have raised again the question as to whether and how the various subclasses differ in their $\gamma$-ray properties.

For a deeper understanding of the fundamental mechanisms at work in these sources it is crucial to address the questions: within the blazar phenomenon which is the physical origin of the difference among BL Lacs and even more 
broadly between BL Lacs and FSRQ? Is it possible to identify continuity among them, with a limited number of physical properties determining the observational characteristics of all blazars?

We address these issues from two sides: a purely observational approach (Fossati et al. 1998) based on complete sub-samples of blazars and (here) a more theoretical approach based on modeling individually the SEDs of all the $\gamma$-ray sources with sufficient available data to constrain their physical parameters. This allows us to derive trends between the physical quantities underlying the correlations between the observed ones.

Several models, still in competition, have been proposed to explain the $\gamma$-ray emission and the overall SED of blazars. Mannheim (1993) proposed that shock-accelerated electrons and protons give origin to two different populations of particles (electrons and electron-positron pairs), responsible of the entire SED through synchrotron emission. In an alternative widely adopted scenario, a single population of electrons is supposed to radiate from the far IR (or even radio) to the UV-soft $\mathrm{X}$-rays by the synchrotron mechanism, and at higher frequencies by the inverse Compton process. In general the observed SEDs require curved spectra steepening at higher frequencies, for both the synchrotron and inverse Compton components. In the $\nu F_{\nu}$ representation of the SEDs each component shows then a peak that in the following will be referred to respectively as the synchrotron and inverse Compton peak. Specific models differ in the adopted geometry (one-zone homogeneous models or inhomogeneous jet models), and in the nature of the target photons which are up-scattered in energy by the inverse Compton process. The target photons could be either synchrotron photons (Maraschi, Ghisellini \& Celotti 1992; Bloom \& Marscher 1993; Ghisellini \& Maraschi 1994), or be produced in the accretion disk (Dermer \& Schlickeiser 1993), or in the broad line region (BLR). The BLR itself can be either illuminated by the disk (Sikora, Begelman \& Rees 1994; Blandford 1993; Blandford \& Levinson 1995), or self-illuminated by the jet (Ghisellini \& Madau 1996). Finally, target photons could be produced by a dusty torus surrounding the blazar nucleus (Wagner et al. 1995). All these different scenarios have been tested on specific sources, but often more than one model can reproduce the same data with similar accuracy (see von Montigny et al. 1997 for 3C 273; Ghisellini, Maraschi \& Dondi 1996 for 3C 279; Comastri et al. 1997 for 0836+710).

Here we examine only two of the leading pictures, namely the synchrotron self-Compton (SSC) and the 'external Compton' (EC) model, in which the main contribution to the target photons is produced outside the $\gamma$-ray emitting region, even if some contribution from the SSC component is always present. Therefore, in the following, with the term "EC" we mean a model in which both the SSC and the EC contributions to the high energy spectrum are considered, while external photons are completely neglected in the SSC model. The SSC and the EC models are applied to all sources with sufficient available data to constrain the models themselves.

Through a search in the literature the multiwavelength overall spectra of $51 \gamma$-loud blazars have been assembled. Even if the vast majority of the data are not simultaneous and the sample is not complete in any respect, they provide a useful template of the SED of different classes of $\gamma$-loud blazars. While the non-simultaneity of the data (except for a few sources) precludes from deriving strong conclusions about specific objects, their large number allows us to study trends in the physical parameters of the models and possible correlations among them and with the observed spectral characteristics of different sub-classes of blazars.

In Section 2 the sample of sources is defined, while in Section 3 we describe the two adopted models, the computing procedures and the 'fit' criteria. The results are presented in Section 4 and discussed in Section 5.

\section{THE SAMPLE}

Two pieces of information have been considered essential for including a source in the present sample:

(i) either detection and estimate of the $\gamma$-ray spectral slope in the EGRET band or detection by the WHIPPLE observatory;

(ii) measured (or lower limit on) redshift.

For all the sources satisfying the above criteria sufficient information at lower frequencies could be found so that the location of both the synchrotron and inverse Compton peaks and the luminosity of each source could be estimated. The resulting 51 sources are listed in Table 1 in the Appendix, together with their redshift, classification and the list of references relative to the data plotted in Fig. 1a-f. The sample includes 14 BL Lac objects and 37 quasars. Among quasars, all core dominated radio sources with flat radio spectra, 16 are HPQ (highly polarized: optical polarization $>3$ per cent), 16 are LPQ (lowly polarized), while for the remaining 5 , labeled NP, polarization measurements were not found.

BL Lacs can be divided in two sub-classes with different broad band spectra according to their radio-X-ray spectral index $\alpha_{\mathrm{RX}}$ (Padovani \& Giommi 1995, Fossati et al. 1998). In fact there is a close correlation between the value of $\alpha_{\mathrm{RX}}$ and the energy of the synchrotron emission peak: for $\alpha_{\mathrm{RX}}>$ 0.75 this is in the IR-optical (LBL: low frequency BL Lac), otherwise in the UV-soft X-ray band (HBL: high frequency BL Lac). According to this definition, we have $10 \mathrm{LBL}$ and 4 HBL.

Note that in our list there are two sources detected by WHIPPLE but not by EGRET, i.e. the two HBL objects Mkn $501(1652+398)$ and 1ES 2344+514.

In Fig. 1a-f, the overall SEDs of all the blazars listed in Table 1 are plotted. Frequencies and luminosities are in the rest frame of the source and are calculated assuming cosmological parameters $H_{0}=50 \mathrm{~km} \mathrm{~s}^{-1} \mathrm{Mpc}^{-1}$ and $q_{0}=0.5$. Fluxes have been dereddened using the absorption values reported in the NED database.

\section{THE MODELS}

\subsection{General assumptions}

The emitting region is assumed to be a sphere (blob) of constant radius $R$, with a homogeneous and tangled magnetic field $B$. Throughout the source relativistic electrons are continuously injected at a rate $Q(\gamma)\left[\mathrm{cm}^{-3} \mathrm{~s}^{-1}\right]$, corresponding to a luminosity $L_{\text {inj }}$ and a compactness $\ell_{\text {inj }} \equiv$ $L_{\mathrm{inj}} \sigma_{\mathrm{T}} /\left(R m_{\mathrm{e}} c^{3}\right)$, where $\sigma_{\mathrm{T}}$ is the Thomson scattering cross 


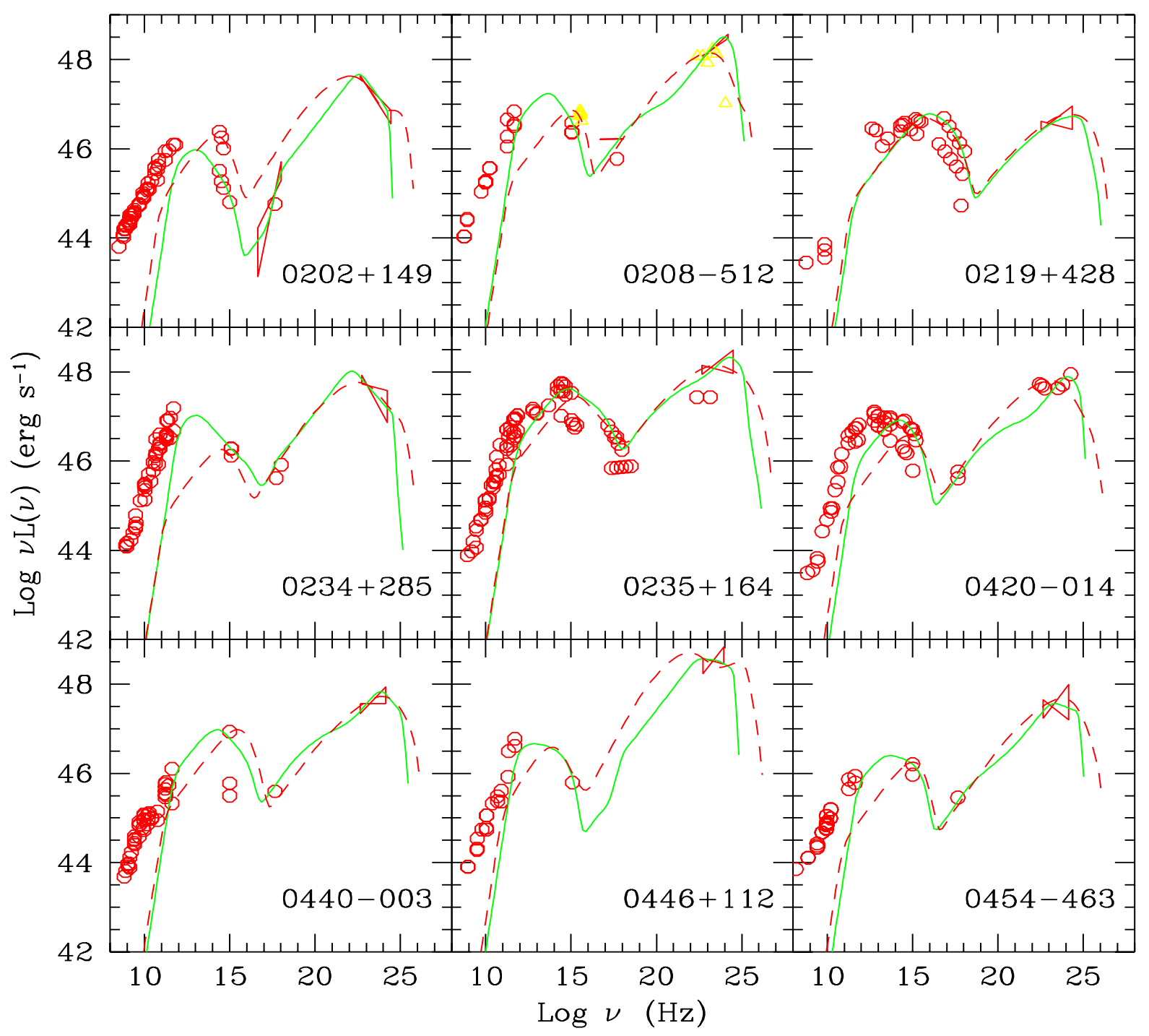

Figure 1. a. Spectral energy distributions (in $\nu L(\nu)$ ) of the $51 \gamma$-ray loud sources. The broad band spectra have been assembled from data in the literature (the complete list of references is given in Table 1). In parenthesis the rescaling factors used for graphical purpose are indicated. SED from the SSC and EC models are superposed to the data, as dashed and solid line, respectively. The model parameters are reported in Table 2, in the Appendix. For 0716+714 a redshift $z=0.3$ has been adopted.

section. This power is assumed to be entirely converted into radiation. The injected particles are distributed in energy as a power-law of slope $s\left[Q(\gamma)=Q_{0} \gamma^{-s}\right]$, between $\gamma_{\min }$ and $\gamma_{\max }$.

The blob moves with a bulk velocity $\beta c$, corresponding to a Lorentz factor $\Gamma$, at an angle $\theta$ with respect to the line of sight. The Lorentz transformation of the specific intensity is thus given by $I(\nu)=\delta^{3} I^{\prime}(\nu / \delta)$, where $\delta=[\Gamma(1-\beta \cos \theta)]^{-1}$ is the Doppler factor. For simplicity (see below), we always assume $\theta \sim 1 / \Gamma$, resulting in $\delta \sim \Gamma$. In the following of this section, unless otherwise specified, all quantities are measured in the blob comoving frame.

We consider a stationary situation, that is we determine the particle equilibrium distribution and the spec- trum of the emitted radiation self-consistently, assuming that the timescale over which the particles reach equilibrium is shorter than that over which the injection mechanism changes. We neglect particle escape and adiabatic expansion.

\subsection{The particle distribution}

The equilibrium particle distribution $N(\gamma)\left(\mathrm{cm}^{-3}\right)$ is determined by the stationary solution of the continuity equation

$$
N(\gamma)=\frac{\int_{\gamma}^{\gamma_{\max }}[Q(\gamma)+P(\gamma)] d \gamma}{\dot{\gamma}}
$$




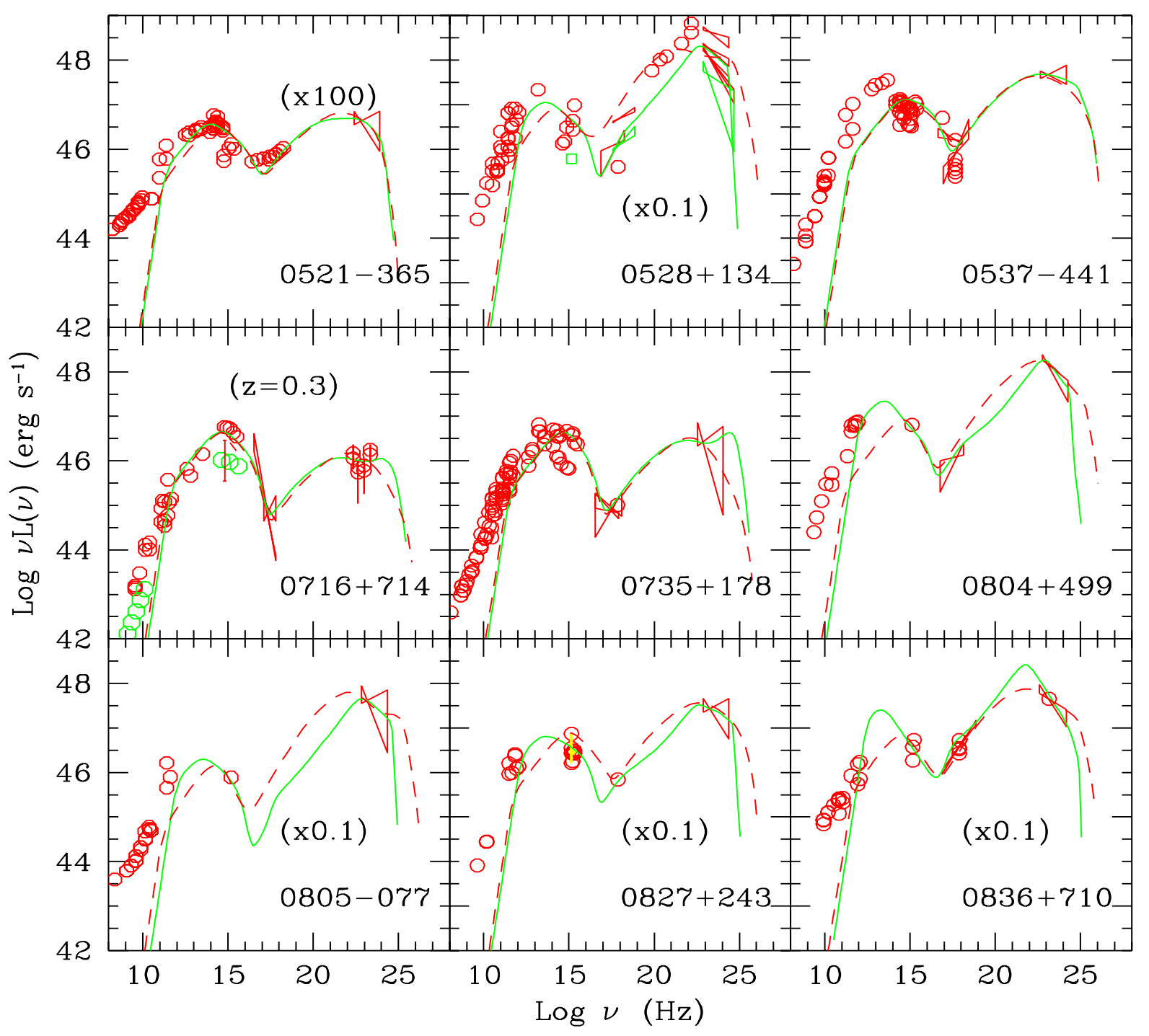

Figure 1. b Same as Fig. 1a

where $\dot{\gamma}$ is the cooling term and $P(\gamma)$ is the rate of electronpositron pair production. The only important mechanism for pair production is photon-photon collisions, the rate of which is calculated according to the prescriptions given in e.g. Ghisellini (1989).

$\dot{\gamma}$ takes into account the following cooling mechanisms:

(i) synchrotron emission: $m_{\mathrm{e}} c^{2} \dot{\gamma}_{\mathrm{s}}=(4 / 3) \sigma_{\mathrm{T}} c \gamma^{2} U_{\mathrm{B}}$, where $U_{\mathrm{B}}=B^{2} /(8 \pi)$ is the magnetic energy density;

(ii) inverse Compton emission: $m_{\mathrm{e}} c^{2} \dot{\gamma}_{\mathrm{C}}=(4 / 3) \sigma_{\mathrm{T}} c \gamma^{2} U_{\mathrm{r}}$, where $U_{\mathrm{r}}$ is the radiation energy density. Since the radiation spectrum extends to high energies, the scattering process has to be calculated by means of the Klein-Nishina cross section. For simplicity, we approximate it with a step function equal to the Thomson cross section for frequencies $x \equiv h \nu /\left(m_{\mathrm{e}} c^{2}\right) \leq(3 / 4) / \gamma$, and zero otherwise. This implies that the radiation energy density effectively involved in the inverse Compton cooling depends on the electron energy

$$
U_{\mathrm{r}}(\gamma)=m_{\mathrm{e}} c^{2} \int_{0}^{3 /(4 \gamma)} U(x) d x
$$

The continuity equation is solved numerically, with an iterative approach, as described in Ghisellini (1989). The numerical treatment is necessary because of the high nonlinearity of the processes involved: $N(\gamma)$ depends on the radiation spectrum (because of the inverse Compton cooling term and the pair production rate), which in turn is determined by $N(\gamma)$.

When the Klein-Nishina and pair production effects can be neglected, the solution of equation 1 is trivial: 1) for injection indices $s>2$, we have a broken power law: $N(\gamma) \propto \gamma^{-2}$ up to $\gamma_{\min }$ and $N(\gamma) \propto \gamma^{-s-1}$ above. In this case $\gamma_{\min }$ can be identified with the crucial parameter $\gamma_{\text {peak }}$, i.e. the Lorentz factor of the electrons emitting at the peaks of 


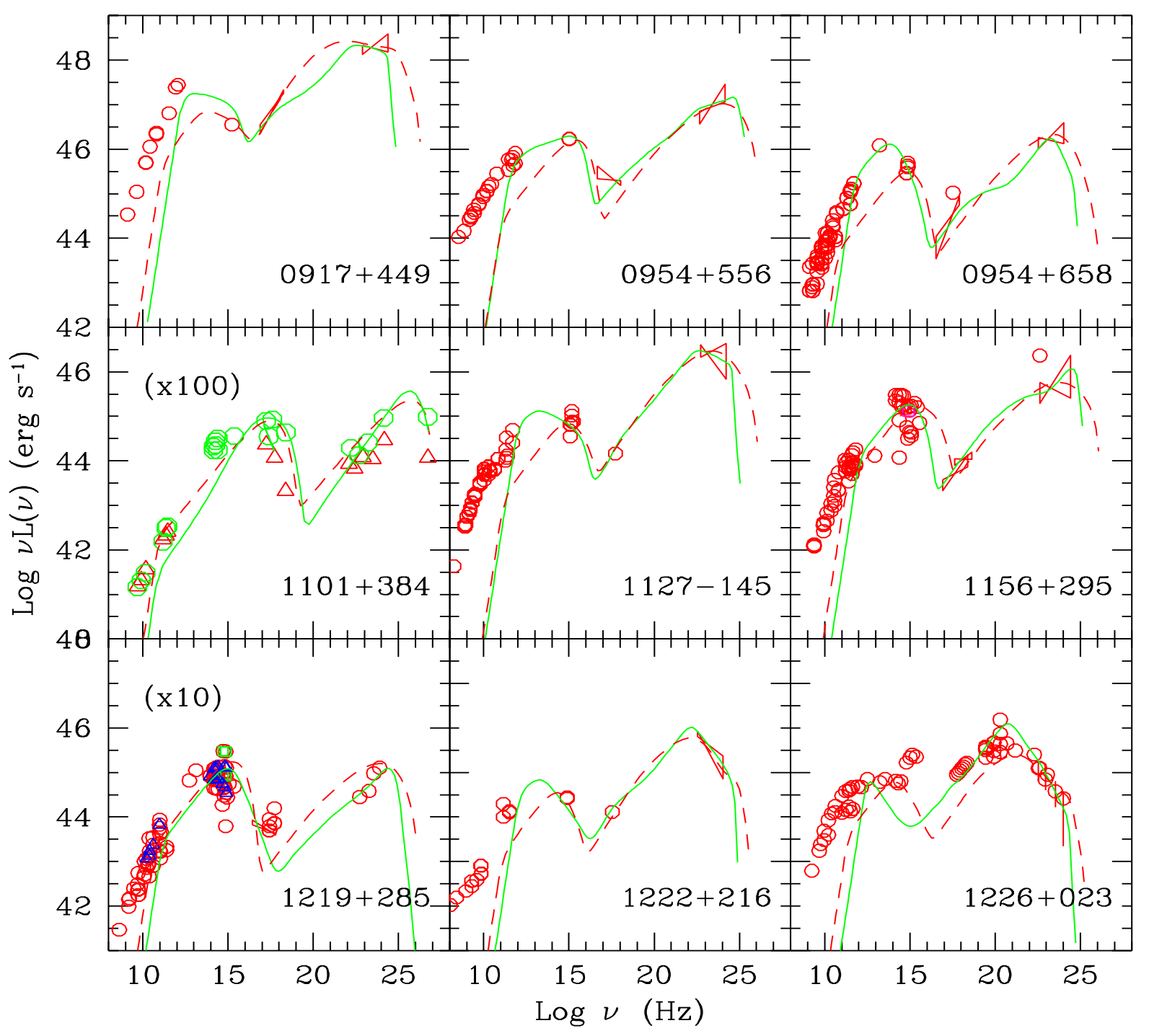

Figure 1. c Same as Fig. 1a

the synchrotron and inverse Compton components; 2) if $1<s<2$ we have the same solutions for $N(\gamma)$, but in this case $\gamma_{\text {min }}<\gamma_{\text {peak }}<\gamma_{\max }$, since the spectral index of the radiation emitted by particles above $\gamma_{\min }$ is flatter than unity; 3) if $s<1$ the lower limit of the integral in equation 1 becomes unimportant, yielding $N(\gamma) \propto \gamma^{-2}$ in the entire energy range, except for $\gamma$ close to $\gamma_{\max }$.

Note that the assumption of constant radius and no escape tends to overestimate the particle distribution at the lowest energies, where these effects are potentially more important than radiative cooling (if the overall compactness is much less than unity). This has no effect on the synchrotron spectrum, which is self-absorbed at low frequencies, and has no effect on the observable SSC spectrum, mainly made by high energy electrons. In the case of the EC model, instead, the $\mathrm{X}$-ray spectrum is made by the sum of the EC and SSC components, and therefore the X-ray flux and spectrum can depend on the details of the low energy particle distribution if the EC component dominates. Then in these cases the calculated X-ray spectrum could be flatter than what derived here.

\subsection{Target photons}

$U_{\mathrm{r}}(\gamma)$ includes the contribution from the radiation both produced internally (by synchrotron and self-Compton emission) and externally to the blob.

The latter is assumed to be distributed as a (diluted) blackbody, peaking at a frequency $x_{\text {ext }} \equiv h \nu_{\text {ext }} /\left(m_{\mathrm{e}} c^{2}\right)$ between $5 \times 10^{-5}$ and $2 \times 10^{-4}$ (in the rest frame of the blob). The exact value depends on the radiation mechanism responsible for the external field and the bulk Lorentz factor of the blob. The assumption of a blackbody spectral distribution is merely for ease of calculation. For instance, in the 


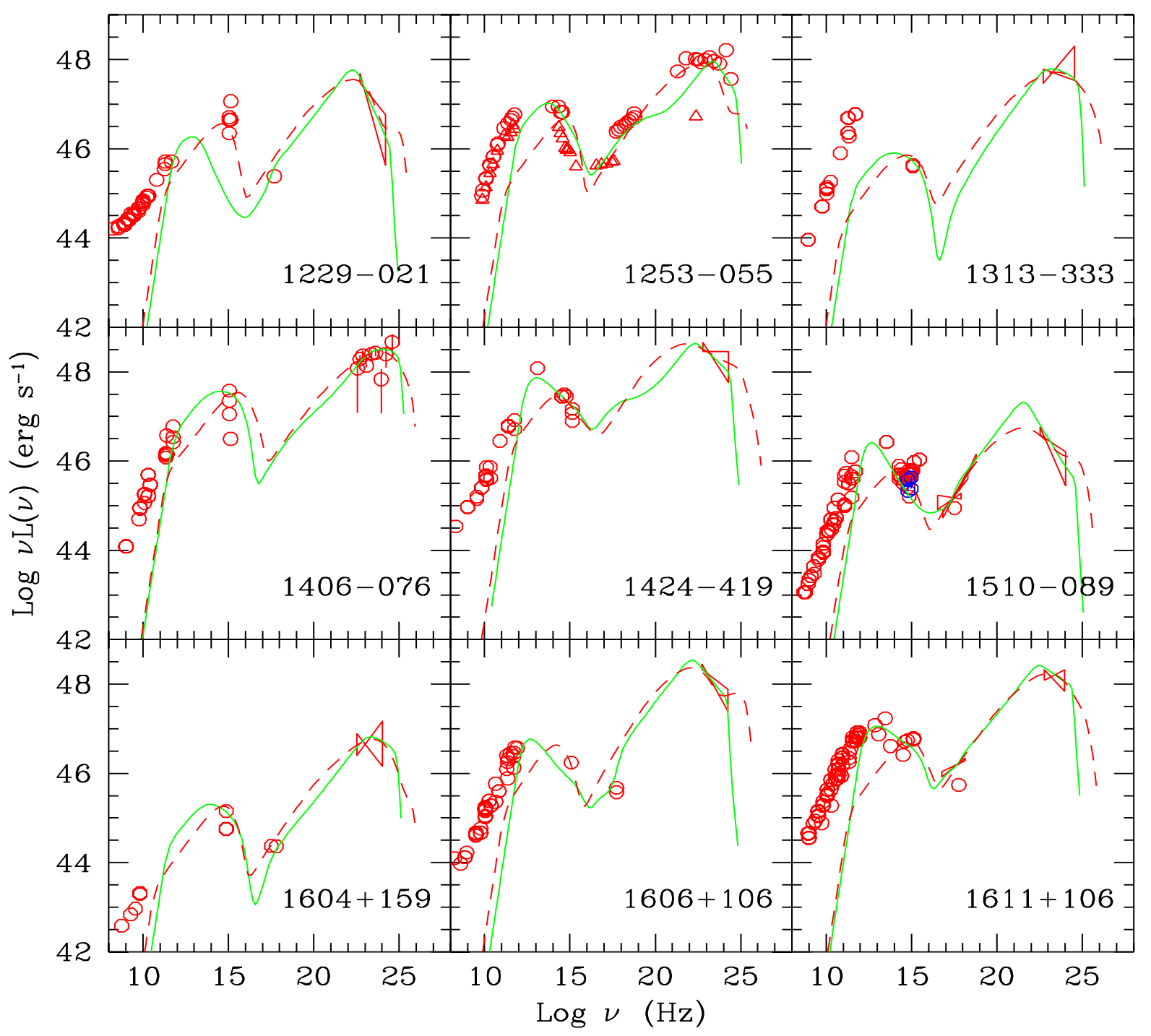

Figure 1. d Same as Fig. 1a

case of external radiation dominated by the broad emission line photons, an observer in the comoving frame of the blob would see a complex spectrum, not isotropic (blueshifted in the forward direction and redshifted in the opposite one): even a single, monochromatic line would be transformed into a peaked, but extended, spectrum.

Consequently, a peaked distribution can approximate the case of externally produced photons distributed in lines, independently of the origin of the photoionizing continuum: we can treat the cases of disk-illuminated as well as jetilluminated BLR. On the other hand, this assumption can mimic the effect of an external scattering medium only if the illuminating continuum is narrowly distributed in frequency (e.g. radiation produced by an accretion disk), but it is not satisfactory for a scattering medium illuminated by the jet (which produces a more extended spectrum).

For a direct comparison with the value of the compact- ness in injected electrons, $\ell_{\text {inj }}$, we assume that also the external radiation can be characterized by an 'effective compactness' $\ell_{\text {ext }}$, defined as

$$
\ell_{\mathrm{ext}}=\frac{\sigma_{\mathrm{T}} R U_{\mathrm{ext}}}{m_{e} c^{2}}
$$

where $U_{\text {ext }}$ is the radiation energy density (of the external radiation) as seen in the comoving frame, and is therefore amplified by a factor $\Gamma^{2}$ with respect to the same quantity measured in the frame of the observer.

As already mentioned, this external field is not isotropic in the comoving frame (see Dermer 1995). However both for simplicity and because of the uncertainty in the origin and therefore in the angular distribution of the external radiation, we assume an isotropic pattern for $\ell_{\text {ext }}$ (in the comoving frame). With this approximation also the inverse Compton radiation is isotropically distributed in this frame, 


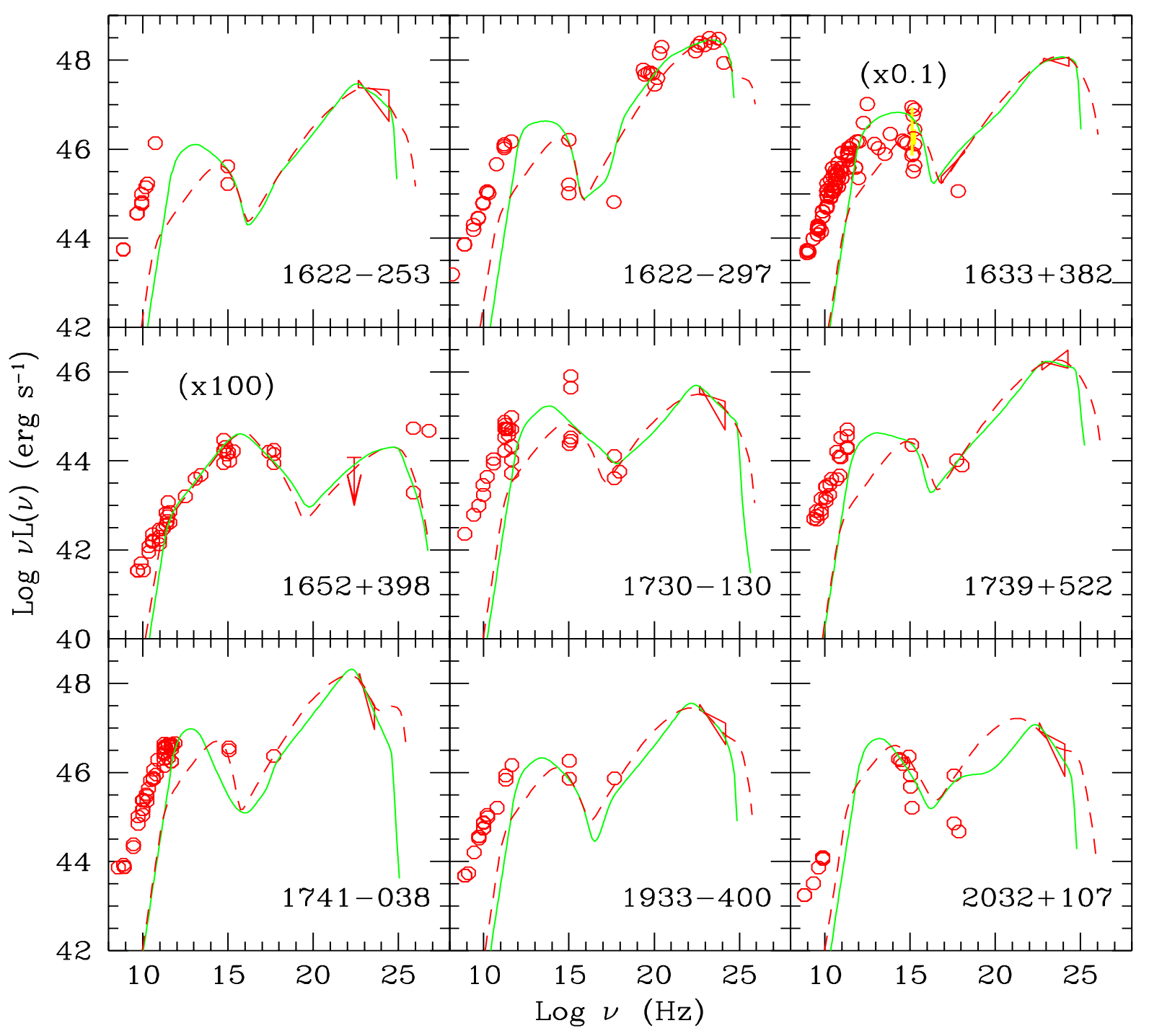

Figure 1. e Same as Fig. 1a

and subject to the same Lorentz transformation as the synchrotron and self-Compton emission.

The uncertainty related to the latter assumption can be estimated comparing the two extreme cases of the Compton flux emitted assuming (in the comoving frame) an isotropic seed photon distribution and the case of soft photons distributed only along the jet axis. Assume for simplicity that in both cases the seed photons are monochromatic, at the frequency $\nu_{0}^{\prime}$. The total power emitted by an electron of energy $\gamma m_{e} c^{2}$ is $P=\sigma_{T} c U_{\mathrm{rad}}^{\prime}\left[\gamma^{2} \int(1-\beta \cos \phi)^{2} d \Omega /(4 \pi)-1\right]$ (see e.g. Rybicky \& Lightman 1979), where $\phi$ is the angle between the incoming photon and the electron velocity vector and $U_{\text {rad }}^{\prime}$ is the energy density of the seed photons. The viewing angle $\theta=1 / \Gamma$ corresponds to the aberrated angle $\theta^{\prime}=90^{\circ}$ : at this angle, the power received in the isotropic case is $P_{\text {iso }}=(4 / 3) \sigma_{T} c U_{\text {rad }}^{\prime}$, while the power received in the monodirectional case $\left(\phi=90^{\circ}\right)$ is
$P_{\text {mono }}=\sigma_{T} c U_{\text {rad }}^{\prime}\left(\gamma^{2} / 2-1\right)$. The ratio $P_{\text {mono }} / P_{\text {iso }}$ for large $\gamma$ is therefore equal to $3 / 8$. The corresponding ratio between the scattered frequencies is equal to $3 / 2$.

\subsection{Observational constraints}

We require that the model parameters, besides giving a good description of the broad band SED, also satisfy additional constraints, regarding the observed variability timescales $t_{\mathrm{var}}$ and the amount of Doppler boosting. In fact, as commonly observed for the optical-UV and $\gamma$-ray emission of blazars, the minimum variability timescale must be as short as a day, or a fraction of a day. This corresponds to demand

$$
R \lesssim c t_{\mathrm{var}} \frac{\delta}{1+z}
$$

with $t_{\mathrm{var}} \sim 1$ day. 


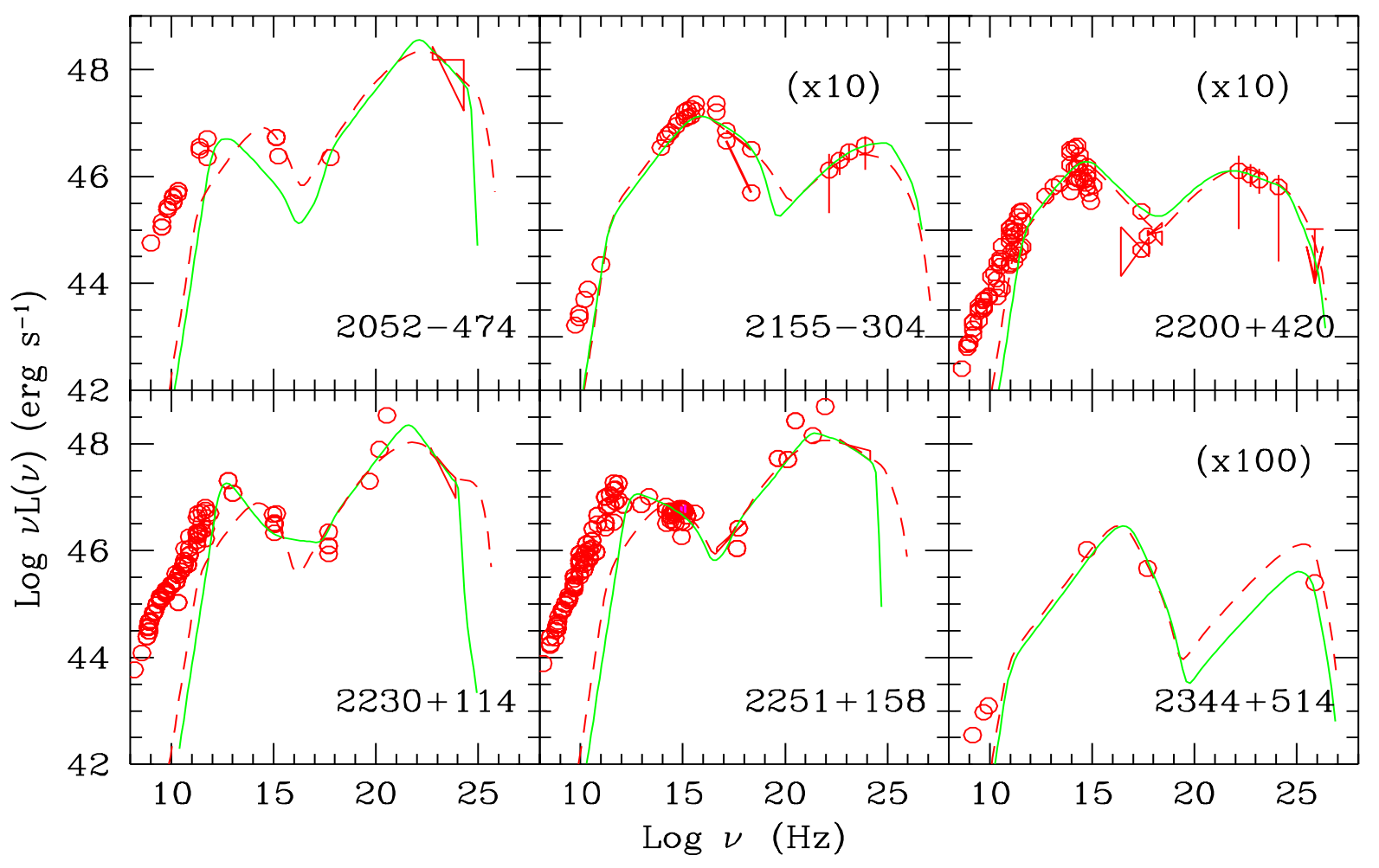

Figure 1. f Same as Fig. 1a

Furthermore, the Doppler factor is constrained not to exceed a value of $20-25$, to be consistent with the observed superluminal speeds.

A third requirement, which is not imposed a priori but has to be satisfied in all cases, concerns the amount of pair production. As discussed in Ghisellini \& Madau (1996), the $\gamma$-ray emitting region must be thin to the high energy radiation, otherwise it inevitably leads to overproduction of $\mathrm{X}$-rays. The line of the argument is as follows: if a substantial fraction of the power emitted in the $\gamma$-ray band gets absorbed in photon-photon collisions, the pairs created (which are relativistic) radiate their energy in other bands by synchrotron and inverse Compton emission. In particular this results in a copious production of $\mathrm{X}$-rays, with a luminosity of the same order as that in $\gamma$-rays. Since the importance of the pair production process is measured by the compactness, the transparency requirement translates into an upper limit to the allowed values of $\ell_{\text {inj }} \lesssim 1$ (see Dondi \& Ghisellini 1995).

Summarizing, for the pure SSC model 7 input parameters are required, namely: $R, B, \Gamma, \ell_{\mathrm{inj}}, s, \gamma_{\min }, \gamma_{\max }$. If the inverse Compton scattering on external photons (EC model) is included, two more parameters are required $\ell_{\text {ext }}$ and $x_{\text {ext }}$ (i.e. a total of 9 ).

However, if the slope of the injected electron distribution is steep $(s>2)$, which is the case for most sources, the exact value of $\gamma_{\max }$ becomes energetically unimportant and is practically irrelevant in the comparison with spectral data. Furthermore, if the external radiation is constituted of broad line photons, the value of $x_{\text {ext }}$ is constrained in a very narrow energy range.

In conclusion, even if there are formally nine free input parameters, $\gamma_{\max }$ is relatively unimportant, $x_{\text {ext }}$ is tightly limited and constraints apply to the possible values of $R, \delta$ and $\ell_{\text {inj. }}$.

Given the number of free parameters, a key question concerns the uniqueness of the 'fits'.

From an observational point of view, we have already mentioned the most critical quantities, namely the energy and luminosity of the two spectral peaks, which determine the global spectral shape. Furthermore, the optical-to-Xrays and the $\mathrm{X}$-ray-to- $\gamma$-ray spectral indices and the three limits discussed above (on $R, \delta, \ell_{\text {inj }}$ ) also constrain the parameters.

In particular, as shown by Ghisellini et al. (1996), in the SSC scenario all the parameters are strongly constrained by the frequencies of the synchrotron and the self-Compton peaks, and by the corresponding powers. These allow to uniquely determine $B, \delta$ and $\ell_{\text {inj }} . R$ and $\ell_{\text {inj }}$ have then to satisfy the above constraints.

In the EC scenario, the further free parameter $\ell_{\text {ext }}$ could be in principle be constrained by the observed soft photon component (e.g. emission line intensities). Although we choose not to assume a priori the origin of the external soft photon field, however this should at least contain the contribution of photons produced in the broad line region. The addition of $\ell_{\text {ext }}$ as a free parameter makes the choice of $\delta$ not unique.

Independently of our assumption of selecting values of $\delta$ 

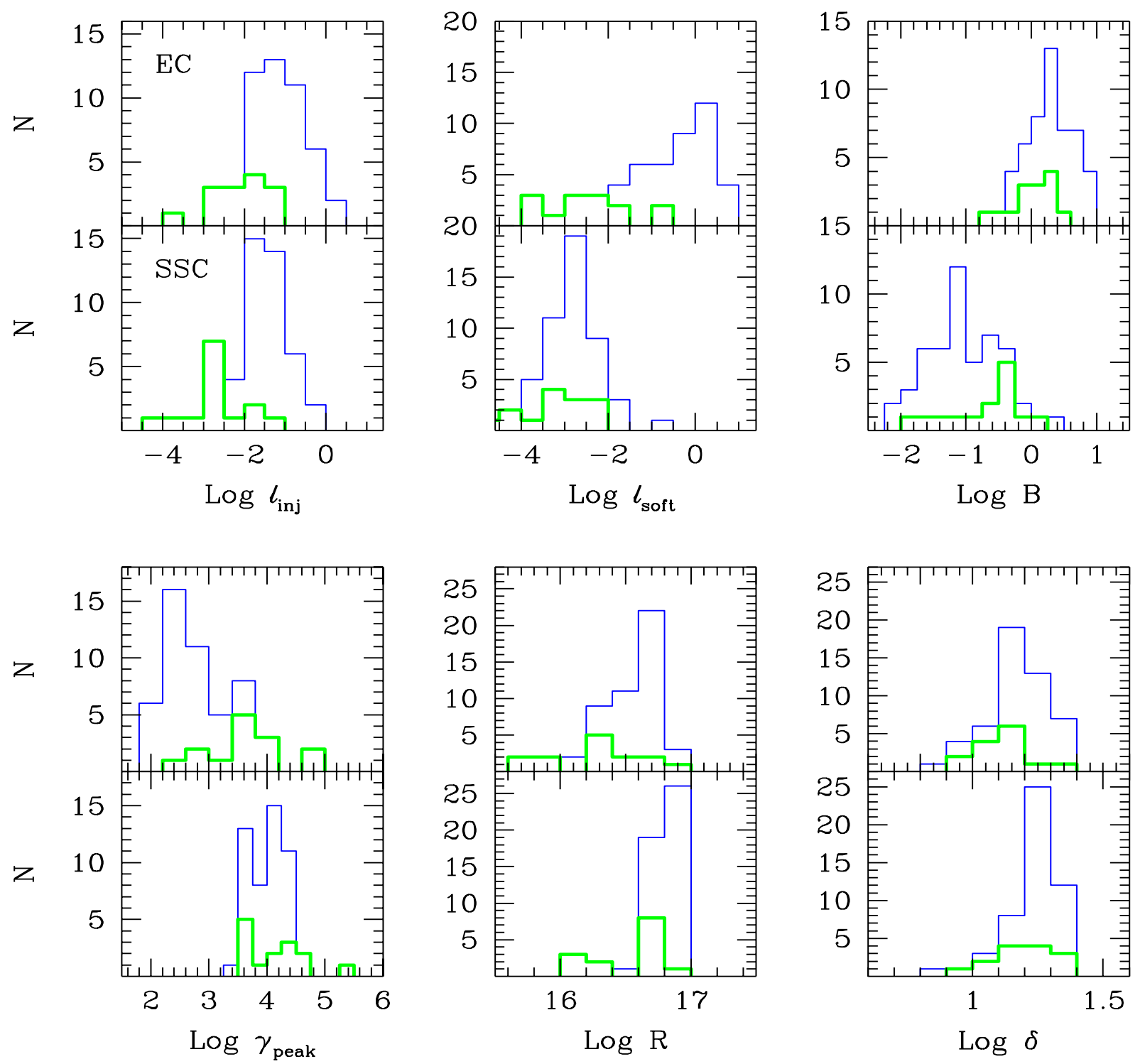

Figure 2. The histograms show the distributions of the parameters of the fits for the EC (upper panels) and the SSC (lower panels) models for all sources. Thick lines represent BL Lac objects. $\ell_{\text {soft }}$ is $\ell_{\text {ext }}$ in the case of the EC model and $\ell_{\text {syn }}$ (compactness of the synchrotron radiation) in the case of the SSC model

consistent with the observed superluminal speeds, we have therefore examined the consequences of allowing arbitrary values of $\delta$. In principle, one can obtain good fits with, e.g., $\delta \sim 100$ and small values of $\ell_{\text {inj }}\left(\propto \delta^{-4}\right)$. But since $\ell_{\text {ext }} \propto$ $\Gamma^{2} \sim \delta^{2}$, the external photons become important as targets in collisions with very high energy $\gamma$-rays, with the result of overproducing X-rays (see $\S 3.4$ and Ghisellini \& Madau 1996). Assuming instead a small value of $\ell_{\text {ext }}$ corresponds to limit the broad line radiation to an implausibly small contribution. We therefore conclude that also the EC model is well constrained.

The main source of uncertainty on the model parameters is given by the incomplete and poor spectral coverage of several sources, which does not allow to determine with accuracy the observational constraints, most critically the peak of the synchrotron emission. Another source of uncertainty regards the presence of other spectral components. Our single-zone and homogeneous models cannot reproduce the spectrum at frequencies below the far IR, since at these frequencies the model spectrum is self-absorbed. Other emitting regions, of greater dimensions, are necessary to fit the radio band. If these also emit in the IR and optical bands, they could contribute to the synchrotron spectrum, possibly shifting its peak at a frequency different from the one corresponding to the $\gamma$-ray emitting region.

As far as the actual fitting procedure is concerned, it should be pointed out that we try to reproduce collections of data that rarely are simultaneous, for sources whose variability is a defining property. Note also that the spectral coverage differs widely from object to object. On one hand 
this makes it difficult to determine a method to estimate the goodness of 'fit' other than the visual inspection (with all its limits). On the other hand, as mentioned in the introduction, the main goal of our study is not to model specific blazars, but to unveil possible trends using a large number of sources. In this sense, although the model parameters relative to a specific source are not 'objectively' found (as it would be for a best fit determined through a statistical $\chi^{2}$ test) nevertheless they represent a reasonable description of the spectral properties of each of the blazar in the sample.

\section{RESULTS}

The spectral distributions derived from the SSC and EC models which better describe the SEDs are superposed to the data in Fig. 1a-f. On the basis of these 'fits' both models can be acceptable for basically all sources. As mentioned in the Introduction, the EC model includes the contribution of the SSC component, i.e. the inverse Compton flux is calculated assuming, as seed photon flux, the sum of the internal synchrotron photons and the externally produced photons. As a rule, the EC component is always dominating at the highest frequencies ( $\gamma$-ray band), and often it also dominates in the $\mathrm{X}$-ray band. Note however that in some sources, as 0208-512, 0420-014, 0440-003, 0735+178, $0954+658,1156+295,1253-055$ and $2032+107$, the X-ray band is mainly produced by the SSC component even in the EC model.

In Table 2 (in the Appendix) the input parameters for all the fits are reported. The most remarkable difference between the two sets of parameters (SSC vs EC) is the relatively smaller value of the magnetic field in the SSC model. This has to be expected, since in order to reproduce the large ratios of inverse Compton to synchrotron luminosities, the SSC model requires a small magnetic energy density, while this constraint is relaxed in the EC scenario.

Although at first sight it seems difficult to discriminate between the two models, at least for FSRQ the parameters derived in the SSC scenario argue in favour of the EC model. In fact, let us consider the typical quantities required by the SSC model: $\delta \sim 20, \ell_{\text {inj }} \sim 0.03, B \sim 0.05 \mathrm{G}, R \sim 10^{17} \mathrm{~cm}$. These imply a compactness in synchrotron radiation $\ell_{\text {syn }} \sim$ $3 \times 10^{-3}$. Consequently, inverse Compton scattering on broad line photons is unimportant if the external radiation energy density $U_{\text {ext }}$ (as seen in the comoving frame) is less than the synchrotron one, i.e.:

$$
L_{\mathrm{BLR}}<\frac{m_{\mathrm{e}} c^{3} \ell_{\mathrm{syn}}}{\sigma_{\mathrm{T}} R \Gamma^{2}} R_{\mathrm{BLR}}^{2} \simeq 3 \times 10^{42} R_{\mathrm{BLR}, 18}^{2} \quad \mathrm{erg} \mathrm{s}^{-1},
$$

where the above typical parameters have been used and $R_{\mathrm{BLR}}=10^{18} R_{\mathrm{BLR}, 18} \mathrm{~cm}$. This limit on $L_{\mathrm{BLR}}$ is certainly not observationally satisfied in FSRQ (e.g. Celotti, Padovani \& Ghisellini 1997). For BL Lacs the situation is ambiguous. While the absence of observable emission lines in most BL Lacs suggests that the SSC process can dominate on the EC one, (weak) broad emission lines have been occasionally observed in some LBL (e.g. BL Lac itself, Vermeulen et al. 1995, Sitko \& Junkkarinen 1985; PKS 0537-441, Stickel, Fried \& Kühr 1993), sometimes exceeding the above limit. And indeed in some cases the inclusion of an external radiation component yields a better broad band fit. Therefore in the following the discussion is focused on the results of the EC scenario. or HBL the different parameters derived in the SSC and EC models can be considered an indication of the allowed range of values and, in particular, the external radiation (e.g emission line luminosity) required by the EC fit can be taken as an upper limit.

Fig. 2 shows the distributions of values of the model parameters within the EC (upper panels) and the SSC (lower panels) scenarios. The thick solid lines correspond to the results for BL Lacs. In the EC case, BL Lac objects almost always form the left tail of the distributions, being characterized by smaller compactness, magnetic field and slightly smaller degree of beaming. On the contrary, comparable dimensions $R$ and greater value of $\gamma_{\text {peak }}$ are required by the EC fits of BL Lacs with respect to FSRQ. In the SSC case the required $\gamma_{\text {peak }}$ is limited in a narrow range, without a clear distinction between BL Lacs and FSRQ, while BL Lacs are characterized by a larger average value of the magnetic field.

As a consequence of the constraints imposed on the Doppler factor and the variability timescales, the distributions of $\delta$ and $R$ span less than one order of magnitude each, with $R \sim 10^{16-17} \mathrm{~cm}$. On the contrary the other (intrinsic) quantities are spread over much larger ranges of values, with the external photon compactness covering the wider interval of about 5 decades.

The injected particle energy distribution is highly different from source to sources (see Table 2), in shape, compactness and (rather low) maximum energy $\gamma_{\max } m_{\mathrm{e}} c^{2}$, thus not requiring a very finely tuned injection/acceleration mechanism.

\subsection{Correlations}

The main goal of this work is to determine trends and correlations among physical quantities which can shed light on the relationship among different sub-classes of blazars and ultimately on the processes at work in these objects.

We have found that the most interesting quantity to investigate links among adopted and derived model parameters is the Lorentz factor at the break of the electron distribution $\gamma_{\text {peak }}$, which determines the location of both the synchrotron and the Compton peaks, and therefore largely determines the shape of the SED. The other important parameters controlling the SED are the ratio of the Compton to synchrotron powers, i.e. the Compton dominance $L_{\mathrm{C}} / L_{\mathrm{syn}}$, the power (or the corresponding compactnesses) injected in the form of electrons (which in our model corresponds to the radiated power), and the power in the external photon component. The results of linear correlations involving these quantities are shown in Figs. 3 and 4 and their statistical significance is reported in Table 3 in the Appendix. For completeness, we list in Table 4 (in the same Appendix) also the results of the linear correlations in the case of the SSC model.

Let us consider the results of the correlations:

$\dagger$ Note that given the high values of the Doppler factors derived from the fits, the assumption $\theta \sim 1 / \Gamma$ is satisfied. The only exception is $0521-365$, which only requires $\delta \sim 1.4$ 

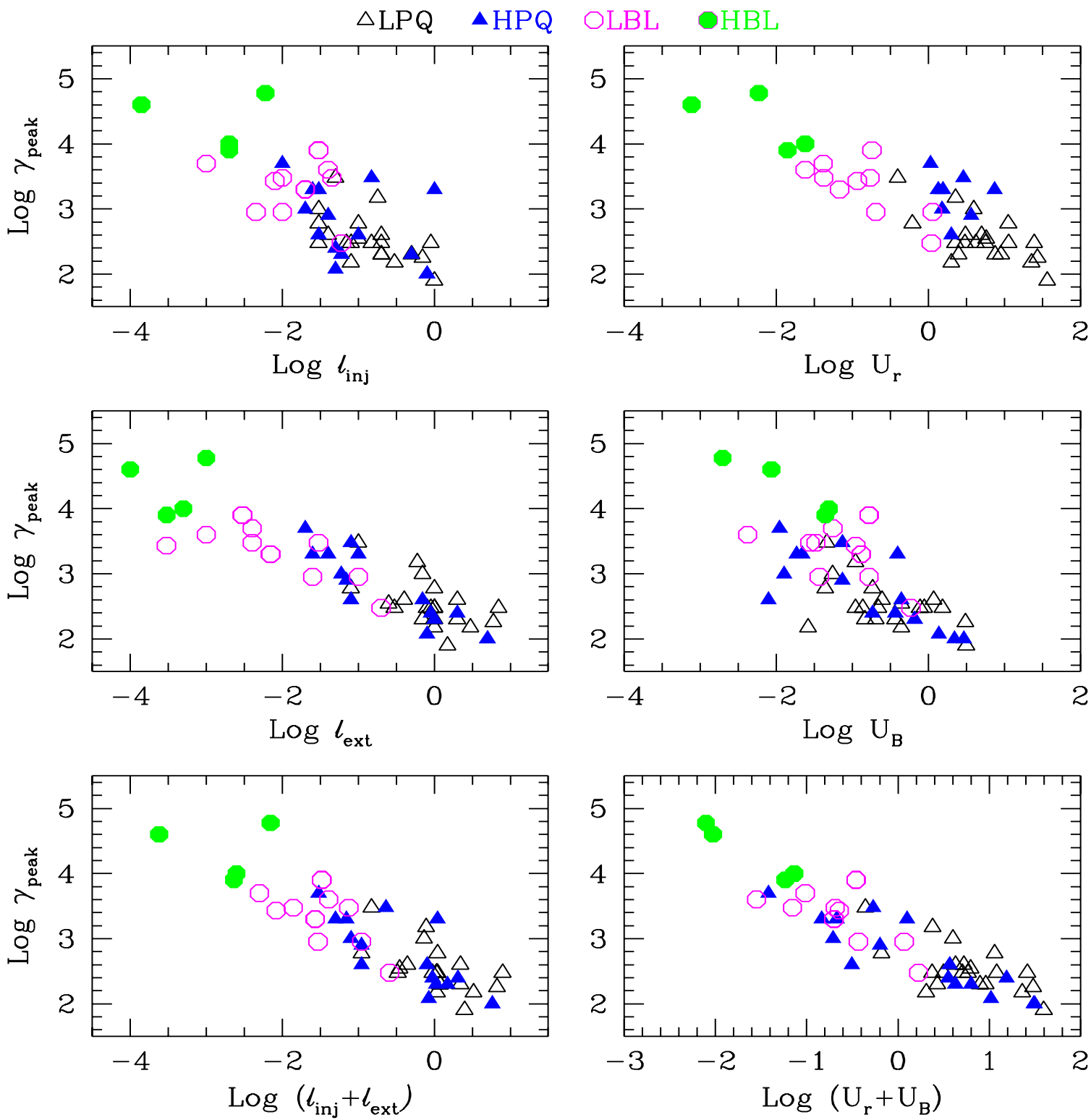

Figure 3. $\gamma_{\text {peak }}$ (from the EC model) is plotted against some other, intrinsic, parameters. The statistical significance of the correlations is reported in Table 3, in the Appendix.

(i) $\gamma_{\text {peak }}$ - Strong correlations are present between $\gamma_{\text {peak }}$ and $\ell_{\text {ext }}, \ell_{\text {inj }}$ and the energy densities in radiation $U_{\mathrm{r}}$ and magnetic field $U_{\mathrm{B}}$, both for the whole sample and for the FSRQ sub-sample. In particular, a very strong linear correlation is found between $\gamma_{\text {peak }}$ and the total energy density, with a dependence $\gamma_{\text {peak }} \propto\left(U_{\mathrm{r}}+U_{\mathrm{B}}\right)^{-0.6}$. The same trend appears from the correlation of $\gamma_{\text {peak }}$ with $\ell_{\text {ext }}+\ell_{\text {inj }}$. It should be pointed out that these correlations are not, or at most only partly, induced by an observational selection effect: there would not be bias against detecting sources with either high values of $\gamma_{\text {peak }}$ and $\left(U_{\mathrm{r}}+U_{\mathrm{B}}\right)$ or viceversa. Furthermore the significance of the correlations (i.e. their small spread) can be taken as a posteriori indication of the tightness of the observational constraints imposed on the model parameters. Note that HBL, LBL and quasars are located along a sequence. (ii) $L_{\mathrm{C}} / L_{\mathrm{syn}}$ - The Compton dominance correlates with $\gamma_{\text {peak }}, \ell_{\text {ext }}, \ell_{\text {inj }}$, and $\nu_{\text {peak }}^{\text {obs }}$, the latter being the observed peak frequency of the modeled synchrotron emission. It also correlates with the observed (beamed) power $L_{\mathrm{inj}}^{\mathrm{obs}}=L_{\mathrm{inj}} \delta^{4}$, while only a weak correlation exists between $L_{\mathrm{C}} / L_{\mathrm{syn}}$ and the magnetic field intensity. The statistical significance of all these correlations is higher when considering the entire blazar sample, while weakens when the subsamples of BL Lacs and FSRQ are considered separately. Again, note that in all cases BL Lacs are 'separated' from FSRQ, with HBL at the extremes and some LBL smoothly overlapping with FSRQ.

(iii) $\ell_{\text {ext }}$ vs $\ell_{\text {inj }}$ - A significant linear correlation is present when FSRQ are considered, while (most) BL Lacs show a relative deficiency in the external photon component with respect to this trend (see Fig. 5). 

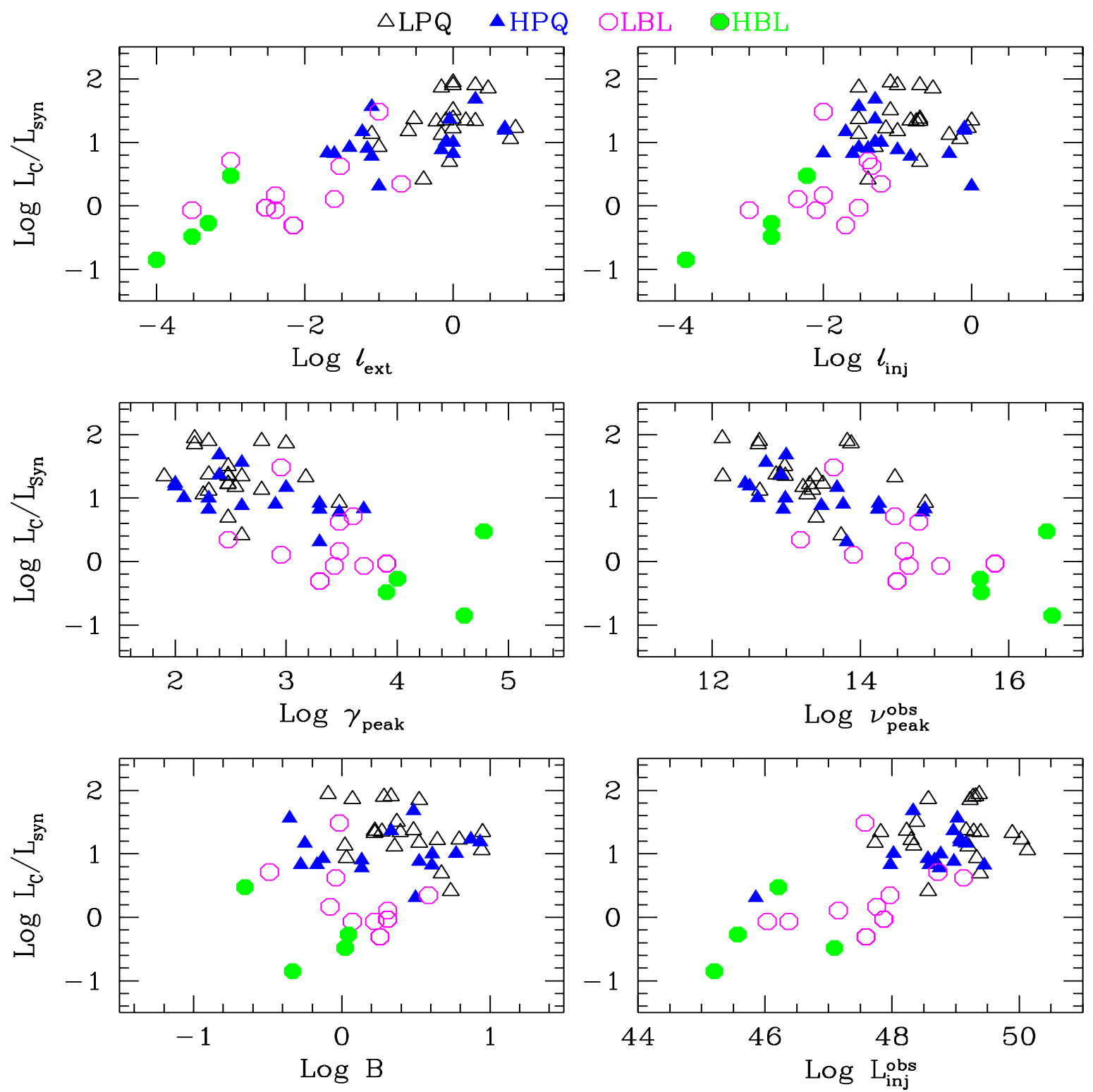

Figure 4. The Compton dominance $L_{\mathrm{C}} / L_{\mathrm{syn}}$ as derived from the EC model is plotted against some other (both intrinsic and 'observable') parameters. The statistical significance of the correlations is given in Table 3 .

To further investigate the correlations among the various quantities described above, we ran a principal component analysis (PCA) program on the correlation matrix. Briefly, the PCA is a method to describe a multidimensional ensemble of correlated parameters, by defining a new coordinate system in which each successive coordinate direction defined by the eigenvectors, explains as much of the remaining variance in the data is possible. PCA reduces the number of relevant components and the remaining should represent more basic parameters than the original ones. (see e.g. Boroson and Green 1992 for an application). We choose to present the PCA done with the 6 most important parameters of the fits: $\ell_{i n j}, \ell_{e x t}, \delta, \gamma_{\text {peak }}, B$ and $R$.

The results of the analysis are presented in Table 6 of the Appendix which lists the most significant eigenvectors in terms of their projection upon the original 6 quantities. At the top of each column the percentage variance accounted for by the eigenvectors is given.

The first eigenvector accounts for about $45 \%$ of the total variance and is dominated by the contribution of the two compactnesses and the magnetic field energy density which anti-correlate (see above) with $\gamma_{\text {peak }}$. This eigenvector could be associated with the total power of the source. The largest contribution to the second eigenvector comes from $\delta, R$ and $\gamma_{\text {peak }}$, while the only relevant projection on the third one is due to $\gamma_{\text {peak }}$.

Given that the Doppler factor $\delta$ and the blob dimension $R$ are not completely independent quantities (see section 3.4), the PCA analysis points to $\gamma_{\text {peak }}, \ell_{\text {inj }}$ and $\ell_{\text {ext }}$ as fundamental variables in explaining the formation of the blazar SED, and confirms the results found through the linear regression analysis. 


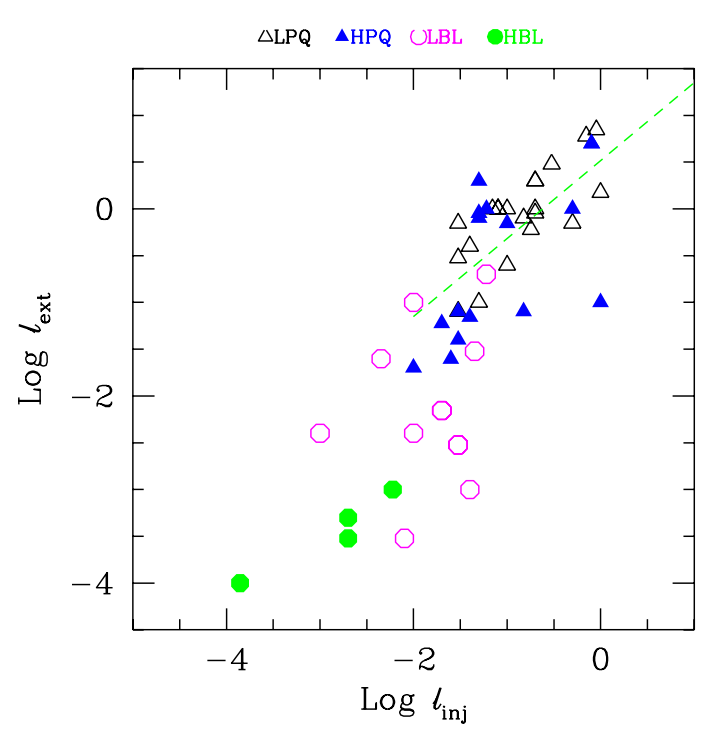

Figure 5. The relation between the compactnesses in external photons $\ell_{\text {ext }}$ and injected power $\ell_{\text {inj }}$. The line represents the bestfit linear correlation for FSRQ only.

\section{DISCUSSION AND CONCLUSIONS}

\subsection{Generalities}

We examined and reproduced the broad band spectral properties of a sample of $51 \gamma$-ray loud blazars, in terms of synchrotron and inverse Compton emission from a homogeneous (one-zone) model. This gives a reasonable good description of the observations at frequencies greater than typically $\sim 10^{11} \mathrm{~Hz}$. The radio emission is expected to be produced by less compact regions on larger jet scales.

On the basis of the 'fits', it is difficult to determine whether the seed field for the inverse Compton scattering is mostly provided by synchrotron or external photons. However, as pointed out by Sikora et al. (1994), the presence of broad emission lines, together with the found values of $\delta$, make photons produced externally to the emitting jet unavoidably important. For this reason we consider the parameters derived in the EC scenario as the most likely for all quasars and some BL Lacs.

Strong correlations have been found among the physical parameters derived from the EC model.

Of particular physical interest is the strong correlation between the energy of electrons emitting at the peak of the observed spectra and the total energy density present in the emitting region, $\gamma_{\text {peak }} \propto\left(U_{\mathrm{r}}+U_{\mathrm{B}}\right)^{-0.6}$. One way to explain this is to assume that $\gamma_{\text {peak }}$ is the result of a competition between the radiative cooling and the (re-)acceleration process, i.e. $\dot{\gamma}_{\text {acc }}\left(\gamma_{\text {peak }}\right) \sim \dot{\gamma}_{\text {cool }}\left(\gamma_{\text {peak }}\right)$. The typical emitting electron would in this case be quickly accelerated up to the energy where cooling is important, while only a few particles would be accelerated at higher energies. The found correlation would then imply that the (re-)acceleration process is almost independent of both the energy density in the region (both in radiation and in magnetic field) and the energy of the particles, since $\dot{\gamma}_{\text {cool }}\left(\gamma_{\text {peak }}\right) \propto \gamma_{\text {peak }}^{2}\left(U_{\mathrm{r}}+U_{\mathrm{B}}\right) \sim$ const. In addition, the injected particle distribution does not require characteristic shape and/or maximum energy. We postpone the discussion of this interesting result to future work; here we would only like to mention the 'hot jet' model put forward by Sikora et al. (1997), in which the balance between heating and cooling can lead to a formation of a peak in the electron energy distribution.

We found another strong correlation between $\gamma_{\text {peak }}$ and the Compton dominance $L_{\mathrm{C}} / L_{\mathrm{syn}}$. On one side this simply confirms and quantifies, from a different perspective, the observational trends pointed out by e.g. Fossati et al. (1998) on the relation between the dominance of the Compton $/ \gamma-$ ray emission and the energy of the peaks of the two spectral components for complete samples of blazars. On the other side, it suggests that this link can be simply interpreted as the consequence of a change in the radiation energy density of the external field. An increase in the latter in fact leads to an increase in the particle Compton cooling and therefore both to a decrease in $\gamma_{\text {peak }}$ and a relative increase in the $\gamma$-ray luminosity. Once again we stress, as discussed in the next Section, that different sub-classes of blazars are located in different areas of this correlation.

As expected from the above correlations $\gamma_{\text {peak }}$ is also (inversely) related to the power injected in the form of relativistic emitting particles.

As presented in Fossati et al. (1998), the ratio of the frequency of the Compton $\left(\nu_{\mathrm{C}}\right)$ to the synchrotron $\left(\nu_{\mathrm{syn}}\right)$ peak is compatible with being approximately constant. Our results are in agreement with these findings, despite the relatively wide range spun by $\gamma_{\text {peak }}(\sim 3$ decades for the EC model). In fact in the EC scenario (and in the Thomson regime of the inverse Compton process $), \nu_{\mathrm{C}} / \nu_{\mathrm{syn}} \sim$ $\Gamma \nu_{\text {ext }} / \nu_{\mathrm{B}}$, is independent of $\gamma_{\text {peak }}$ (here $\nu_{\mathrm{B}}=e B /\left(2 \pi m_{\mathrm{e}} c\right)$ is the cyclotron frequency), and the narrow range of values of $B$ found in the EC model can account for the approximate constant ratio of $\nu_{\mathrm{C}} / \nu_{\mathrm{syn}}$.

In the SSC model, instead, it is $\gamma_{\text {peak }}$ which is found in a narrow range (less than 2 decades). In the SSC case, we expect $\nu_{\mathrm{C}} / \nu_{\mathrm{syn}} \sim \gamma_{\text {peak }}^{2}$ (in the Thomson regime), and $\nu_{\mathrm{C}} / \nu_{\mathrm{syn}} \sim \gamma_{\text {peak }}^{-1}$ in the extreme Klein-Nishina regime (in this case $h \nu_{\mathrm{C}} \sim \gamma_{\text {peak }} m_{\mathrm{e}} c^{2}$ ).

\subsection{The blazar unification}

Evidence for continuity in the observed spectral properties of BL Lacs and FSRQ have been recently found by Fossati et al. (1998), by studying complete samples of sub-classes of blazars in different energy bands. Diagrams and quantities derived by Fossati et al. (1998) from either data or their analytical representation turn out to be similar to and consistent with those found in this paper through model fitting. In Fig. 6, the model considered here is applied to the average SEDs derived by Fossati et al. (1998) by binning, according to the radio luminosity, both BL Lacs and FSRQ belonging to complete samples. The parameters of these fits are reported in Table 5 in the Appendix. The fact that the model fits the average SEDs derived from complete blazars samples, with similar parameters and trends as for the $\gamma$-ray blazars, gives us confidence that our results are valid for all blazars.

The main result of the present paper concerns the intrinsic relationship among phenomenologically different classes of blazars, and in particular the evidence for a well 


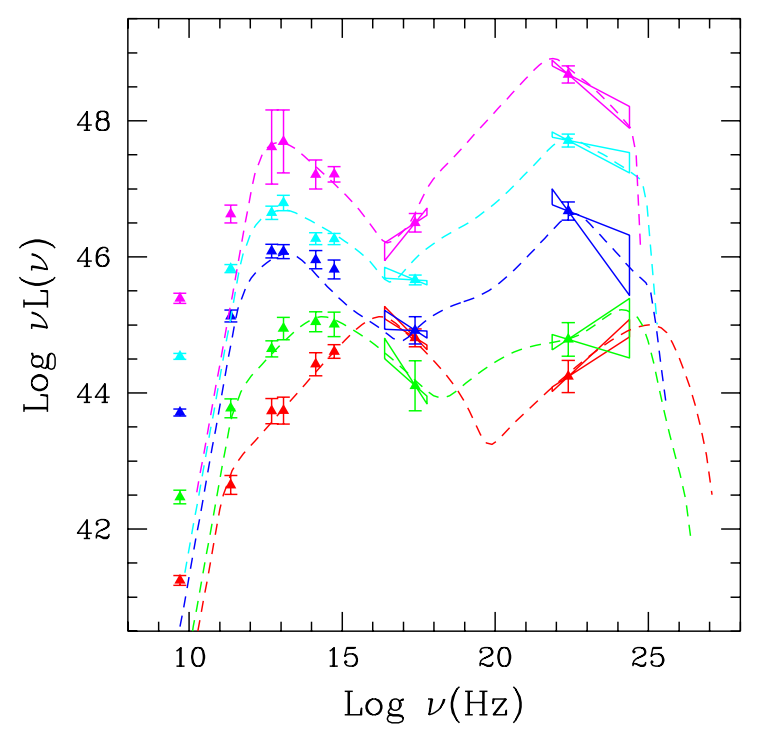

Figure 6. Fits with the EC model of the average SEDs derived by Fossati et al. (1998). BL Lacs and FSRQ belonging to complete samples have been divided in bins accordingly only to their radio luminosity, and the average fluxes in each bin have been computed. The model parameters are reported in Table 5 (Appendix), and are in complete agreement with the parameters determined for the $\gamma$-ray loud sources examined in this work.

defined sequence in the properties of HBL, LBL and FSRQ with increasing importance of an external radiation field: the observed spectral properties of HBL, LBL, HPQ and LPQ can be therefore accounted for by e.g. the increasing role of broad emission line radiation (see also Fig. 6 and Table 5). This in fact dictates the peak energy of the emitting particle distribution and hence the shape of the spectra, thus determining the classification of an object into one of the blazar flavors. The fundamental physical processes occurring in and outside the relativistic jet are instead the same. This is indicated by the correlation between $\ell_{\text {ext }}$ and $\ell_{\text {inj }}$, which seems to 'set in' for more powerful object, from LBL up to the most luminous LPQ (see Fig. 5).

This proposed blazar unifying sequence can be therefore summarized as follows (see the schematic sketch in Fig. 7):

(i) HBL are sources characterized by the lowest intrinsic power and the weakest external radiation field (no or weak emission lines). Consequently the cooling is less dramatic and particles can be present with energies high enough to produce synchrotron emission extending to soft X-ray energies and $\mathrm{TeV}$ radiation through the SSC process. Being the inverse Compton cooling ineffective, the Compton dominance is expected to be small;

(ii) LBL are intrinsically more powerful than HBL and in some cases the external field can be responsible for most of the cooling. The stronger cooling limits the particle energy implying that the synchrotron and inverse Compton emission peak at lower frequencies, in the optical and $\mathrm{GeV}$ bands, respectively, with a larger Compton dominance parameter;

(iii) FSRQ represent the most powerful blazars, where the contribution from the external radiation to the cooling is the

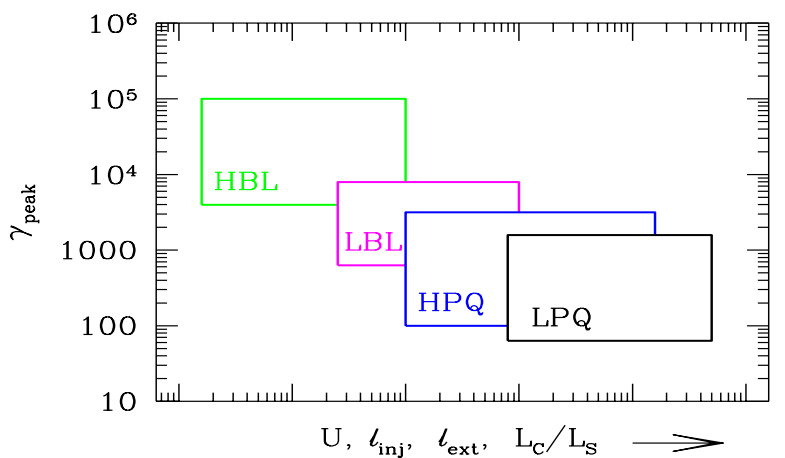

Figure 7. Schematic representation of the proposed unifying scheme: the sequence HBL, LBL, HPQ, LPQ corresponds to an increase in the external radiation field, the total energy density and the injected power. These in turn result in a decrease of $\gamma_{\text {peak }}$ and an increase in the Compton dominance.

greatest. The emission by synchrotron and EC cannot extend at frequencies larger than the IR and $\mathrm{MeV}-\mathrm{GeV}$ bands and the $\gamma$-ray radiation completely dominates the radiative output. Within this class, there is an hint of a further subdivision between low and high polarization objects, with a tendency for LPQ to be more extreme (lower values of $\gamma_{\text {peak }}$ and larger values of $\left(U_{\mathrm{r}}+U_{\mathrm{B}}\right), \ell_{\text {inj }}$ and so on).

The correlations among the different quantities ensure that the knowledge of one of them allows to estimate the entire spectral energy distribution, and also the probable classification of the object. This is of course of great relevance for the study at high energies of those blazars not detected so far in the $\gamma$-ray band and the consequences on their variability patterns and duty cycles. Finally, the above findings have to be taken into account when considering the absorption of high energy radiation by the diffuse background fields as well as the estimates on the blazar contribution to the $\gamma-$ ray background.

In the currently most accepted unification schemes for radio-loud sources, weak and powerful blazars are the beamed counterparts of Fanaroff-Riley type I (FR I, Fanaroff \& Riley 1974) and type II (FR II) radio galaxies, respectively. Indeed continuity in the properties of blazars along a power sequence has been suggested by Maraschi \& Rovetti (1994), Sambruna et al. (1996) and Fossati et al. (1997) on the basis of statistical arguments. Within this frame, the blazar sequence would therefore manifest itself in several observational properties, including the total source power, the luminosity in emission lines, the extended radio power, the dominance of $\gamma$-rays over the other spectral components and the broad band shape of the SED (see Fig. 7). We therefore provide evidence for the unification of all radio-loud sources and suggest a deeper physical understanding for it, based on the total power generated in the very central engine of these spectacular sources.

\section{ACKNOWLEDGMENTS}

Thanks are due to the Italian MURST (Annalisa Celotti, GF) and the Institute of Astronomy PPARC Theory Grant 
(Annalisa Celotti) for financial support. Andrea Comastri acknowledges financial support from the Italian Space Agency under contract ARS-96-70 This research has made use of the NASA/IPAC extragalactic database (NED), which is operated by the Jet Propulsion Laboratory, California Institute of Technology, under contract with the National Aeronautic and Space Administration.

\section{REFERENCES}

Adam G., 1985, A\&AS, 61, 225 (A85)

Aller H.D, Aller M.F., Latimer G.E., Hodge, P.E., 1985, ApJS, 59, 513 (Al85)

Bersanelli M., Bouchet P., Falomo R., Tanzi E.G., 1992, AJ, 104, 28 (Be92)

Bertsch D.L. et al., 1993, ApJ, 405, L21 (Be93)

Biermann P.L., Kuhr H., Snyder W.A., Zensus J.A., 1987, A\&A, 85, 9 (Bi87)

Blandford R.D., 1993, in Friedlander M., Gehrels N., Macomb D.J., eds, Proc. CGRO AIP 280. New York, p. 533

Blandford R.D., Levinson A., 1995, ApJ, 441, 79

Bloom S.D., Marscher A.P., 1991, ApJ, 366, 16 (BM91)

Bloom S.D., Marscher A.P., 1993, in Friedlander M., Gehrels N., Macomb D.J., eds, Proc. CGRO AIP 280. New York, p. 578

Bloom S.D., Marscher A.P., Gear W.K., Terasranta H., Valtaoja E., Aller H.D., Aller M.F., 1994, AJ, 108, 398 (BM94)

Boroson T.A. \& Green R., 1992, ApJS, 80, 109

Bozyan E.P., Hemenway P.D., Argue A.N., 1990, AJ, 99, 1421 (Bo90)

Bregman J.N., Glassgold A.E., Huggins P.J., Kinney A.L., 1985, ApJ, 291, 505 (B85)

Breslin A.C. et al., 1997, IAUC 6592 (B97)

Brinkmann W., Siebert J., Boller T., 1994, A\&A, 281, 355 (B94)

Brinkmann W., Siebert J., Reich W., Furst E., Reich P., Voges W., Trumper J., Wielebinski R., 1995, A\&AS, 109, 147 (B95)

Cappi M., Comastri A., Molendi S., Palumbo G.C.C., Della Ceca R., Maccacaro T., 1994, MNRAS, 271, 438

Catanese M. et al., 1997a, ApJ, 480, 562 (Ca97)

Catanese M. et al., 1997b, proceedings of 25th ICRC (Durban), in press (Cat97)

Celotti A., Padovani P., Ghisellini G., 1997, MNRAS, 286, 415

Chini R., Biermann P.L., Gemund H.-P., 1989, A\&A, 221, L3 (Ch89)

Clegg P.E. et al., 1983, ApJ, 273, 58 (C83)

Collmar, W., 1996, in Gamma ray emitting AGN, MPI H -V37-1996 eds. J.G. Kirk, M. Camenzind, C. von Montigny \& S. Wagner, p. 9 (Co97)

Comastri A., Fossati G., Ghisellini G., Molendi S., 1997, ApJ, 480, 534 (C97)

Condon J.J., Hicks P.D., Jauncey D.L., 1977, AJ, 82, 692 (Co77)

Condon J.L., Anderson E., Broderick J.J., 1995, AJ, 109, 2318 (Co95)

Dermer C.D., 1995, ApJ, 446, L63

Dermer C.D., Schlickeiser R., 1993, ApJ, 416, 458
Dingus B.L. et al., 1996, ApJ, 467, 589 (Di96)

Dondi L. \& Ghisellini G., 1995, MNRAS, 273, 583

Edelson R.A., 1994, AJ, 94, 1150 (E94)

Elvis M., Plummer D., Schachter J., Fabbiano G., 1992, ApJS, 79, 331 (E92)

Falomo R., Scarpa R., Bersanelli M., 1994, ApJS, 93, 125 (F94)

Fanaroff B.L., Riley J.M., 1974, MNRAS, 167, 31

Fichtel C.E. et al., 1994, ApJS, 94, 551

Fossati G., Maraschi L., Celotti A., Comastri A., Ghisellini G., 1998, MNRAS, in press

Fossati G., Celotti A., Ghisellini G. \& Maraschi, L., 1997, MNRAS, 289, 136

Friecke K.J., Kollatschny W., Witzel A., 1983, A\&A, 117, 60 (Fr83)

Giommi P., Ansari S.G., Micol A., 1995, A\&AS, 109, 267

Gear W.K. et al., 1994, MNRAS, 267, 167 (G94)

Ghisellini G., 1989, MNRAS, 238, 449

Ghisellini G., Madau P., 1996, MNRAS, 280, 67

Ghisellini G., Maraschi L., 1989, ApJ, 340, 181

Ghisellini G., Maraschi L., 1994, in Fichtel C.E., Gehrels N., Norris J.P., eds, The Second Compton Symp., AIP 304. New York, p. 616

Ghisellini G., Maraschi L., Tanzi E., Treves A., 1986, ApJ, 310, 317 (G86)

Ghisellini G., Maraschi L., Dondi L., 1996, A\&AS, 120, 503

Ghisellini G. et al., 1997, A\&A, in press (G97)

Glass I.S., 1981, MNRAS, 194, 795 (G81)

Hartmann R.C. et al. 1993, ApJ, 407, L41 (Ha93)

Hunter S.D. et al., 1993, A\&A, 272, 59 (H93)

Impey C.D., Neugebauer G., 1988, AJ, 95, 307 (IN88)

Impey C.D., Tapia S., 1988, ApJ, 333, 666 (IT88)

Impey C.D., Tapia S., 1990, ApJ, 354, 124 (IT90)

Kühr H., Witzel A., Pauliny-Toth I.I.K., Nauber U., 1981, A\&AS, 45, 367 (K81)

Landau R., Jones T.W., Epstein E.E., Neugebauer G., Soifer B.T., Werner M.W., Puschell J.J., Balonek T.J., 1983, ApJ, 268, 68 (L83)

Landau R. et al., 1986, ApJ, 308, 78 (L86)

Lawrence A., Rowan-Robinson M., Efstathiou A., Ward M.J., Elvis M., Smith M.G., Duncan W.D., Robson E.I., 1991, MNRAS, 248, 91 (L91)

Lawson A.J., Turner M.J.L., 1997, MNRAS, 288, 920 (LT97)

Ledden J.F., O’Dell S.L., 1985, ApJ, 298, 630 (Le85)

Lin Y.C. et al., 1995, ApJ, 442, 96 (L95)

Lin Y.C. et al., 1996, ApJS, 105, 331 (L96)

Litchfield S.J., Robson E.I., Stevens J.A., 1994, MNRAS, 270, 341 (Li94)

Lorenzetti D., Massaro E., Perola G.C., Spinoglio L., 1990, A\&A, 235, 35 (L90)

McNaron-Brown K. et al., 1995, ApJ, 451, 575 (Mc95)

Macomb D.J. et al., 1995, ApJ, 449, L99 (Ma95)

Macomb D.J. et al., 1996, ApJ, 459, L111 (Erratum) (Ma96b)

Madejski G., Takahashi T., Tashiro M., Kubo H., Hartman R., Kallman T., Sikora M., 1996, ApJ, 459, 156 (Ma96)

Mannheim K., 1993, A\&A, 269, 67

Maraschi L., Rovetti F., 1994, ApJ, 436, 79

Maraschi L., Schwartz D.A., Tanzi E.G., Treves A., 1985, ApJ, 294, 615 (Ma85)

Maraschi L., Ghisellini G., Celotti A., 1992, ApJ, 397, L5

Maraschi L. et al. 1994, ApJ, 435, L91 (Ma94) 
Maraschi L., Fossati G., Tagliaferri G., Treves A., 1995, ApJ, 443,578

Mattox J.R. et al., 1993, ApJ, 410, 609 (M93)

Mattox J.R, Wagner S.J., Malkna M., McGlynn T.A., Schachter J.F., Grove J.E., Johnson W.N., Kurfess J.D., 1997, ApJ, 476, 692 (M97)

Mattox J.R., Schachter J., Molnar L., Hartman R.C., Patnaik A.R., 1997, ApJ, 481, 95

Mukherjee R. et al., 1995, ApJ, 445, 189 (Mu95)

Mukherjee R. et al., 1996, ApJ, 470, 831 (Mu96)

Nolan P.L. et al., 1993, ApJ, 414, 82 (N93)

Nolan P.L. et al., 1996, ApJ, 459, 100 (N96)

Netzer H. et al., 1996, MNRAS, 279, 429 (Ne96)

Padovani P., Giommi P., 1995, ApJ, 444, 567

Perley R.A., 1982, AJ, 87, 859 (P82)

Perlman E.S. et al., 1996, ApJS, 104, 251 (P96)

Petry D. et al., 1996, A\&A, 311, L13

Pian E. et al., 1993, ApJ, 416, 130 (Pi93)

Pian E., Falomo R., Scarpa R., Treves A., 1994, ApJ, 37, 152 (Pi94)

Pian E., Falomo R., Ghisellini G., Maraschi L., Sambruna R.M., Scarpa R., Treves A., 1996, ApJ, 459, 169 (Pi96)

Pica A.J., Smith A.G., Webb J.R., Leacock R.J., Clements S., Gombola P.P., 1988, AJ, 96, 1215 (P88)

Pohl M., Reich W., Schlickeiser R., Reich P., Ungerechts H., 1996, A\&AS, 120, 529 (Po96)

Quinn J. et al., 1996, ApJ, 456, L83 (Q96)

Radecke H.-D. et al., 1995, ApJ, 438, 659 (R95)

Raiteri C.M. et al., 1997, A\&AS, in press (Ra97)

Reuter H.-P. et al., 1997, A\&AS, 122, 271 (R97)

Rieke G.H., Lebofsky M.J., Wiśniewski W.Z., 1982, ApJ, 263, $73(\mathrm{R} 82)$

Rybicki G.B. \& Lightman A.P., 1979, Radiative processes in Astrophysics, J. Wiley \& Sons (New York)

Robson E.I., Gear W.K., Brown L.M.J., Courvoisier T.J.-L., Smith M.G., 1986, Nat, 323, 134 (R86)

Sambruna R.M., Barr P., Giommi P., Maraschi L., Tagliaferri G., Treves A., 1994, ApJS, 95, 371 (Sa94)

Sambruna R.M. et al., 1997, ApJ, 474, 639 (Sa97)

Schonfelder V., 1994, ApJS, 92, 593 (Sh94)

Sikora M., Begelman M.C., Rees M.J., 1994, ApJ, 421, 153

Sitko M.L., Junkkarinen V.T., 1985, PASP, 97, 1158

Sitko M.L., Sitko A.K., 1991, PASP, 103, 160 (Si91)

Smith P.S., Elston R., Berriman G., Allen R.G., Balonek, T.J., 1988, ApJ, 326, L39 (Sm88)

Sreekumar P. et al., 1996, ApJ, 464, 628 (Sr96)

Stacy J.C., Vestrand W.T., Sreekumar P., Bonnell J., Kubo H., Hartman R.C., 1996, A\&AS, 120, 549 (Sta96)

Steppe H., Salter C.J., Chini R., Kraysa E., Brunswig W., Lobato Perez J., 1988, A\&AS, 75, 317 (St88)

Steppe H., Liechti S., Mauersberger R., Kompe C., Brunswig W., Ruiz-Moreno M., 1992, A\&AS, 96, 441 (St92)

Steppe H. et al., 1993, A\&AS, 102, 611 (St93)

Stevens J.A., Litchfield S.J., Robson E.I., Hughes D.H., Gear W.K., Terasranta H., Valtaoja E., Tornikoski M., 1994, ApJ, 437, 91 (S94)

Stickel M., Fried J.W., Kühr H., 1993, A\&AS, 98, 393

Stickel M., Meisenheimer K., Kühr H., 1994, A\&AS, 105, 211 (St94)

Stickel M., Rieke G.H., Kühr H., Rieke M.J., 1996, ApJ, 468, 556 (St96)

Terasranta H. et al., 1989, A\&AS, 75, 317 (T89)
Terasranta H. et al., 1992, A\&AS, 94, 121 (T92)

Thompson D.J. et al., 1993, ApJ, 415, L13 (T93)

Thompson D.J. et al., 1995, ApJS, 101, 259 (T95)

Thompson D.J. et al., 1996, ApJS, 107, 227 (T96)

Tornikoski M., Valtaoja E., Terasranta H., Lainela M. Bramwell D., Botti L.C.L., 1993, AJ, 105, 1680 (To93)

Tornikoski M. et al., 1996, A\&AS, 116, 157 (To96)

Tosti G. et al., 1997, A\&AS, submitted (To97)

Turner M.J.L. et al., 1990, MNRAS, 244, 310 (T90)

Urry M.C., Padovani P., 1995, PASP, 107, 803

Urry C.M., Sambruna R.M., Worrall D.M., Kollgaard R.I., Feigelson E.D., Perlman E.S., Stocke J.T., 1996, ApJS, 463, 424 (U96)

Urry C.M. et al. 1997, ApJ, in press (U97)

Valtaoja E., Lahteenmaki A., Terasranta H., 1992, A\&AS, 95, 73 (V92)

Vermeulen R.C., Ogle P.M., Tran H.D., Browne I.W.A., Cohen M.H., Readhead, A.C.S., Taylor G.B. \& Goodrich R.W., 1995, ApJ, 425, L5

Vestrand W.T., Stacy J.G., Sreekumar P., 1996, ApJ, 454, L93 (V96)

Villata M. et al., 1997, A\&AS, 121, 119 (V97)

von Montigny C. et al., 1995, ApJ, 440, 525 (vM95)

von Montigny C. et al., 1997, ApJ, in press

Wagner S., Sanchez-Pons F., Quirrenbach A., Witzel A., 1990, A\&A, 235, L1 (W90)

Wagner S.J. et al., 1995, A\&A 298, 688

Wagner S.J. et al., 1995, ApJ, 454, L97 (W95)

Wagner S.J. et al., 1996, AJ, 111, 2187 (W96)

Wall J.V., Peacock J.A., 1985, MNRAS, 216, 173 (WP85)

Webb J.R., Smith A.G., Leacock R.J., Fitzgibbons G.L., Gombola P.P., Shepherd D.W., 1988, AJ, 95, 374 (W88)

Webb J.R., Barnello T., Robson I., Hartman R.C., 1995, IAUC 6168 (We95)

Weekes T.C. et al., 1996, A\&AS, 120, 603

Wilkes B.J., Tananbaum H., Worral D.M., Avni Y., Oey M.S., Flanagan J., 1994, ApJS, 92, 53 (Wi94)

Wiren S., Valtaoja E., Terasranta H., Kotilainen J., 1992, AJ, 104, 111 (W92)

Worral D.M., Wilkes B.J., 1990, ApJ, 360, 396 (WW90)

Worral D.M., Giommi P., Tananbaum H., Zamorani G., 1987, ApJ, 313, 596 (W87)

Wright A.E., Wark R.M., Troup E., Otrupcek R., Jennings D., Hunt A., Andcooke D.J., 1991, MNRAS, 251, 330 (W91) 


\section{APPENDIX}

Table 1. List of sources. (1),(2) Source names; (3) redshift; (4) classification: HPQ and LPQ stand for highly and lowly polarized quasars, while NP indicates sources with no polarization measure; HBL, LBL and IBL refer to high, low and intermediate frequency BL Lacs, respectively; (5) references to the data of Fig. 1.

\begin{tabular}{|c|c|c|c|c|}
\hline $\begin{array}{l}\text { Source } \\
(1)\end{array}$ & $\begin{array}{l}\text { Other name } \\
\quad(2)\end{array}$ & $\begin{array}{c}z \\
(3)\end{array}$ & $\begin{array}{c}\text { Class. } \\
(4)\end{array}$ & $\begin{array}{l}\text { Refs. for data } \\
\text { (5) }\end{array}$ \\
\hline $0202+149$ & $4 \mathrm{C} 15.05$ & 0.833 & HPQ & B85, BM94, C97, IT90, K81, NED, St92, St96, vM95 \\
\hline $0208-512$ & PKS & 1.003 & HPQ & Be93, C97, IT88, NED, Sta96, To96 \\
\hline $0219+428$ & $3 \mathrm{C} 66 \mathrm{~A}$ & 0.444 & LBL & C97, Di96, G86, NED, Pi93, Si91, T95, WW90 \\
\hline $0234-285$ & CTD 20 & 1.213 & $\mathrm{HPQ}$ & B95, BM91, E94, K81, NED, P82, St93, T92, vM95 \\
\hline $0235+164$ & $\mathrm{AO}$ & 0.940 & LBL & BM94, E94, G94, H93, K81, Ma96, NED, Pi93, S94, Si91, To96, WW90 \\
\hline $0420-014$ & PKS & 0.915 & HPQ & $\begin{array}{l}\text { BM94, Ch89, Co95, E94, IN88, K81, Li94, NED, Ra97, R95, S94, Si91, } \\
\text { Sm88, To96, WW90 }\end{array}$ \\
\hline $0440-003$ & NRAO 190 & 0.844 & HPQ & Bo90, K81, NED, St88, T95, To93, To96, W87, WP85 \\
\hline $0446+112$ & PKS & 1.207 & $\mathrm{NP}$ & NED, St88, T95, To96, W92 \\
\hline $0454-463$ & PKS & 0.858 & LPQ & B94, Fr83, IT90, K81, NED, To96, vM95, W91, WP85 \\
\hline $0521-365$ & PKS & 0.055 & $\mathrm{HPQ}$ & C97, IN88, NED, Pi93, Pi94, Pi96, T95, To96 \\
\hline $0528+134$ & OG 147 & 2.07 & $\mathrm{LPQ}$ & $\begin{array}{l}\text { B85, BM94, Co77, Co96, E94, IT90, Mc95, Mu96, NED, Po96, R82, R97, } \\
\text { Sa97, V92, WP85 }\end{array}$ \\
\hline $0537-441$ & PKS & 0.896 & LBL & Be92, C97, IN88, K81, L96, Ma85, NED, Pi93, Sa94, WP85, WW90 \\
\hline $0716+714$ & S5 & $>0.3$ & LBL & BM94, C94, E94, G97, IN88, K81, L95, NED, St92, W96 \\
\hline $0735+178$ & PKS & $>0.424$ & LBL & C97, E94, G94, IN88, NED, N96, Pi93, Si91, To96, WW90 \\
\hline $0804+499$ & 0J 508 & 1.433 & HPQ & BM94, C97, NED, St94, vM95 \\
\hline $0805-077$ & PKS & 1.837 & $\mathrm{NP}$ & K81, NED, St93, T95 \\
\hline $0827+243$ & OJ 248 & 2.05 & LPQ & B95, BM94, NED, Ra97, To96, V97, vM95 \\
\hline $0836+710$ & $4 \mathrm{C} 71.07$ & 2.172 & LPQ & BM94, C97, E92, E94, K81, NED, Ra97, T93, W90, W92, WP85 \\
\hline $0917+449$ & $\mathrm{~S} 4$ & 2.18 & LPQ & BM94, C97, E94, NED, St93, T95 \\
\hline $0954+556$ & 4C55.17 & 0.901 & HPQ & BM94, C97, G94, K81, NED, Sr96 \\
\hline $0954+658$ & $\mathrm{~S} 4$ & 0.368 & LBL & C97, G94, IN88, K81, L86, Mu95, NED, St88 \\
\hline $1101+384$ & Mkn 421 & 0.031 & HBL & Ma95, Ma96b \\
\hline $1127-145$ & PKS & 1.187 & LPQ & A85, B94, Bo90, E94, IT90, K81, NED, Sr96, To96 \\
\hline $1156+295$ & $4 \mathrm{C} 29.45$ & 0.729 & $\mathrm{HPQ}$ & $\begin{array}{l}\text { BM94, E94, G94, IN88, Le85, Li94, LT96, NED, Pi93, Ra97, S94, Sa94, } \\
\text { Si91, To96, V97, vM95, We95 }\end{array}$ \\
\hline $1219+285$ & ON 231 & 0.102 & LBL & BM91, C97, E92, E94, IN88, Lo90, NED, Pi93, Si91, To97, vM95, WW90 \\
\hline $1222+216$ & $4 \mathrm{C} 21.35$ & 0.435 & LPQ & B95, NED, Sr96, To96 \\
\hline $1226+023$ & $3 \mathrm{C} 273$ & 0.158 & LPQ & Al85, C83, G94, IN88, K81, L83, Mc95, NED, R86, Sh94, T90 \\
\hline $1229-021$ & PKS & 1.045 & LPQ & K81, NED, P88, Ra97, Sr96, St88, St94, To96, Wi94 \\
\hline $1253-055$ & $3 \mathrm{C} 279$ & 0.538 & $\mathrm{HPQ}$ & Ma94 \\
\hline $1313-333$ & PKS & 1.210 & NP & N96, NED, St88, St92, St94, To96 \\
\hline $1406-076$ & PKS & 1.494 & LPQ & NED, St88, To93, To96, W95 \\
\hline $1424-418$ & PKS & 1.522 & $\mathrm{HPQ}$ & G81, IN88, NED, To96, T96 \\
\hline $1510-089$ & PKS & 0.361 & $\mathrm{HPQ}$ & $\begin{array}{l}\text { C97, E94, G81, G94, IN88, K81, L86, LT97, NED, Pi93, Ra97, Sa94, Si91, } \\
\text { Sm88, Sr96, St93, To96, V97, WP85 }\end{array}$ \\
\hline
\end{tabular}


Table 1. continue

\begin{tabular}{lcccl}
\hline $\begin{array}{l}\text { Source } \\
(1)\end{array}$ & $\begin{array}{c}\text { Other name } \\
(2)\end{array}$ & $\begin{array}{c}z \\
(3)\end{array}$ & $\begin{array}{c}\text { Class. } \\
(4)\end{array}$ & $\begin{array}{l}\text { Refs. for data } \\
(5)\end{array}$ \\
\hline $1604+159$ & 4C15.54 & 0.357 & LBL & GAM95, IN88, Le85, NED, Sr96 \\
$1606+106$ & 4C 10.45 & 1.227 & LPQ & B95, Bi87, BM94, E94, IT90, K81, NED, To96, vM95 \\
$1611+343$ & DA 406 & 1.404 & LPQ & C97, E94, G94, K81, NED, Ra97, vM95 \\
$1622-253$ & PKS & 0.786 & LPQ & N96, NED, St94 \\
$1622-297$ & PKS & 0.815 & LPQ & K81, M97, NED, St93 \\
$1633+382$ & 4C 38.41 & 1.814 & LPQ & BM91, BM94, Bo90, C97, IN88, K81, M93, Ra97, V92, V97, WP85 \\
$1652+398$ & Mkn 501 & 0.055 & HBL & B97, BM91, C97, G94, IN88, L91, Pi93, Q96, Sa94, St88, W92, WW90 \\
$1730-130$ & NRAO 530 & 0.902 & NP & B94, BM91, E94, N96, NED, St88, St93, To96, W88 \\
$1739+522$ & 4C 51.37 & 1.375 & HPQ & B95, BM91, K81, NED, St88, St93, St94, V92, vM95 \\
$1741-038$ & OT-68 & 1.054 & HPQ & B94, E94, G94, K81, NED, St88, St92, St93, St94, To96, vM95 \\
$1933-400$ & PKS & 0.966 & NP & B94, Di96, K81, NED, St94, To96 \\
$2032+107$ & PKS & 0.601 & LBL & Di96, GAM95, NED, WW90 \\
$2052-474$ & PKS & 0.071 & LPQ & B94, IT90, K81, NED, To96, vM95 \\
$2155-304$ & PKS & 0.117 & HBL & U97, V96 \\
$2200+420$ & BL Lac & 0.069 & LBL & BM94, Ca97, E94, IN88, NED, P96, Pi93, S94, St93, To96, U96 \\
$2230+114$ & CTA 102 & 1.037 & HPQ & B94, BM94, E94, F94, IN88, K81, Le85, Mc95, N93, Ne96, Ra97, St93, \\
& & & & T89, To96, W92, Wi94 \\
$2251+158$ & 3C 454.3 & 0.859 & HPQ & Be92, BM91, BM94, C97, E94, G94, Ha93, IN88, K81, Le85, Mc95, \\
& & & & Ne96, NED, Pi93, Ra97, S94, Sm88 \\
$2344+514$ & 1ES & 0.044 & HBL & Cat97, NED, P96 \\
\hline
\end{tabular}


Table 1. continue

\begin{tabular}{|c|c|}
\hline A85: & Adam (1985) \\
\hline B85: & Bregman et al. (1985) \\
\hline B95: & Brinkmann et al. (1995) \\
\hline Be92: & Bersanelli et al. (1992) \\
\hline Bi87: & Biermann et al. (1987) \\
\hline BM94: & Bloom et al. (1994); \\
\hline C83: & Clegg et al. (1983) \\
\hline C97: & Comastri et al. (1997) \\
\hline Cat97: & Catanese et al. (1997b) \\
\hline Co77: & Condon, Hicks \& Jauncey (1977) \\
\hline Co96: & Collmar (1996) \\
\hline E92: & Elvis et al. (1992) \\
\hline F94: & Falomo, Scarpa \& Bersanelli (1994) \\
\hline GAM95: & Giommi, Ansari \& Micol (1995): \\
\hline G86: & Ghisellini et al. (1986) \\
\hline G97: & Ghisellini et al. (1997) \\
\hline Ha93: & Hartmann et al. (1993) \\
\hline IT88: & Impey \& Tapia (1988) \\
\hline K81: & Kühr et al. (1981) \\
\hline L86: & Landau et al. (1986) \\
\hline L95: & Lin et al. (1995) \\
\hline Le85: & Ledden \& O'Dell (1985) \\
\hline Lo90: & Lorenzetti et al. (1990) \\
\hline M93: & Mattox et al. (1993) \\
\hline Ma85: & Maraschi et al. (1985) \\
\hline Ma95: & Macomb et al. (1995) \\
\hline Ma96b: & Macomb et al. (1996) \\
\hline Mu95: & Mukherjee et al. (1995) \\
\hline N93: & Nolan et al. (1993) \\
\hline Ne96: & Netzer et al. (1996) \\
\hline P88: & Pica et al. (1988) \\
\hline Pi93: & Pian et al. (1993) \\
\hline Pi96: & Pian et al. (1996) \\
\hline Q96: & Quinn et al. (1996) \\
\hline R86: & Robson et al. (1986) \\
\hline R97: & Reuter et al. (1997) \\
\hline S94: & Stevens et al. (1994) \\
\hline Sa97: & Sambruna et al. (1997) \\
\hline Si91: & Sitko \& Sitko (1991) \\
\hline Sr96: & Sreekumar et al. (1996) \\
\hline St92: & Steppe et al. (1992) \\
\hline St94: & Stickel, Meisenheimer \& Kuhr (1994) \\
\hline Sta96: & Stacy et al. (1996) \\
\hline T90: & Turner et al. (1990) \\
\hline T93: & Thompson et al. (1993) \\
\hline T96: & Thompson et al. (1996) \\
\hline To96: & Tornikoski et al. (1996) \\
\hline U96: & Urry et al. (1996) \\
\hline V92: & Valtaoja, Lahteenmaki \& Terasranta (1992) \\
\hline V97: & Villata et al. (1997) \\
\hline W87: & Worral et al. (1987) \\
\hline W90: & Wagner et al. (1990) \\
\hline W92: & Wiren et al. (1992) \\
\hline W96: & Wagner et al. (1996) \\
\hline Wi94: & Wilkes et al. (1994) \\
\hline WW90: & Worral \& Wilkes (1990) \\
\hline
\end{tabular}

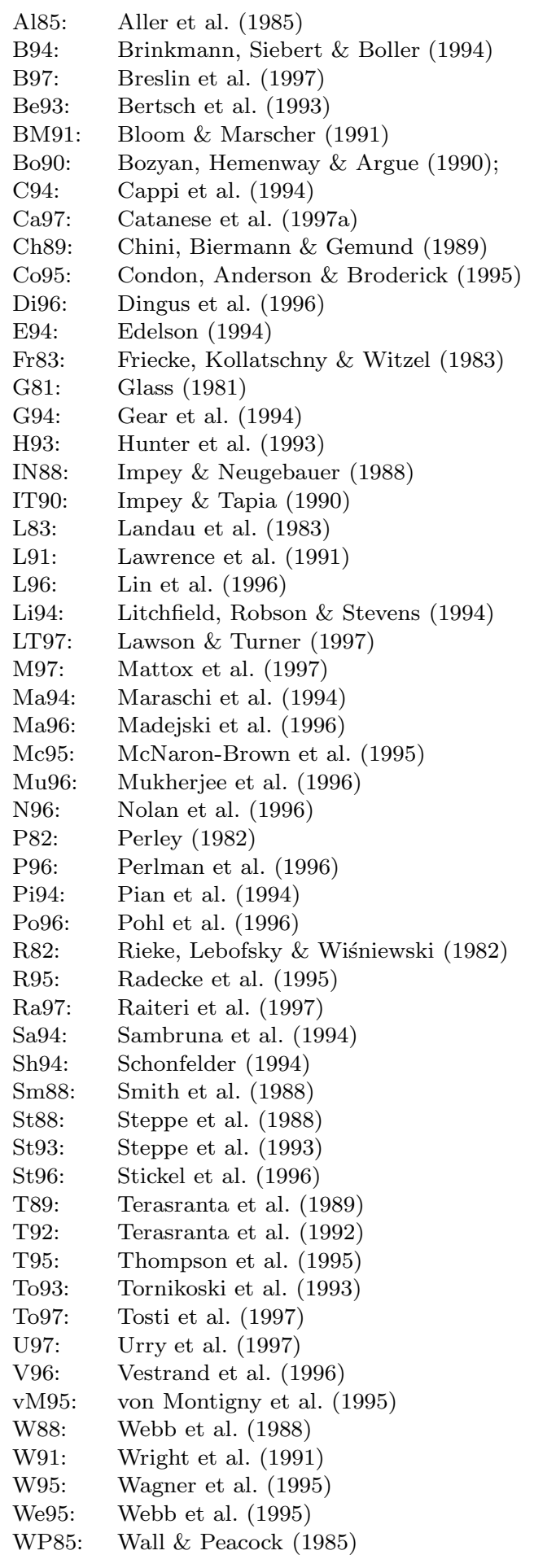


Table 2. The input parameters for the EC and SSC models are reported in the first and second line for each source, respectively. (1) Source name; (2) region size in units of $10^{15} \mathrm{~cm}$; (3), (4) compactnesses in injected particles and external radiation field; (5) maximum energy of the injected particles; (6) energy of the peak of the stationary electron distribution; (7) spectral index of the injected particles; (8) magnetic field intensity (in Gauss); (9) relativistic Doppler factor. The SSC model for 1253-055 requires monoenergetic injection.

\begin{tabular}{|c|c|c|c|c|c|c|c|c|}
\hline $\begin{array}{l}\text { Source } \\
\text { (1) }\end{array}$ & $\begin{array}{c}R / 10^{15} \\
(2)\end{array}$ & $\begin{array}{l}\ell_{\text {inj }} \\
(3)\end{array}$ & $\begin{array}{l}\ell_{\text {ext }} \\
(4)\end{array}$ & $\begin{array}{c}\gamma_{\max } \\
(5)\end{array}$ & $\begin{array}{c}\gamma_{\text {peak }} \\
(6)\end{array}$ & $\begin{array}{c}s \\
(7)\end{array}$ & $\begin{array}{c}B \\
(8)\end{array}$ & $\begin{array}{c}\delta \\
(9)\end{array}$ \\
\hline \multirow[t]{2}{*}{$0202+149$} & 30 & 0.05 & 2 & $3.0 \mathrm{e} 3$ & $2.5 \mathrm{e} 2$ & 3.0 & 3.040 & 14 \\
\hline & 80 & 0.01 & - & $4.0 \mathrm{e} 4$ & $1.0 \mathrm{e} 4$ & 3.3 & 0.026 & 18 \\
\hline \multirow[t]{2}{*}{$0208-512$} & 70 & 0.02 & 0.06 & $7.0 \mathrm{e} 3$ & $1.0 \mathrm{e} 3$ & 3.8 & 0.563 & 23 \\
\hline & 50 & 0.08 & - & $3.0 \mathrm{e} 4$ & $1.5 \mathrm{e} 4$ & 2.0 & 0.106 & 16 \\
\hline \multirow[t]{2}{*}{$0219+428$} & 20 & 0.03 & $3 e-3$ & $1.0 \mathrm{e} 5$ & $8.0 \mathrm{e} 3$ & 2.4 & 2.040 & 13.5 \\
\hline & 40 & 0.01 & - & $2.0 \mathrm{e} 5$ & $1.0 \mathrm{e} 4$ & 2.4 & 0.590 & 15 \\
\hline \multirow[t]{2}{*}{$0234-285$} & 40 & 0.06 & 1 & $1.0 \mathrm{e} 4$ & $2.0 \mathrm{e} 2$ & 2.9 & 4.080 & 16 \\
\hline & 50 & 0.08 & - & $6.0 \mathrm{e} 4$ & $1.0 \mathrm{e} 4$ & 3.0 & 0.067 & 13 \\
\hline \multirow[t]{2}{*}{$0235+164$} & 50 & 0.05 & 0.03 & $8.0 \mathrm{e} 4$ & $3.0 \mathrm{e} 3$ & 3.0 & 0.912 & 20 \\
\hline & 60 & 0.02 & - & $3.0 \mathrm{e} 5$ & $1.0 \mathrm{e} 4$ & 2.9 & 0.215 & 21 \\
\hline \multirow[t]{2}{*}{$0420-014$} & 50 & 0.03 & 0.04 & $1.0 \mathrm{e} 4$ & $2.0 \mathrm{e} 3$ & 3.5 & 0.745 & 16 \\
\hline & 80 & 0.01 & - & $8.0 \mathrm{e} 4$ & $1.5 \mathrm{e} 4$ & 3.0 & 0.034 & 20 \\
\hline \multirow[t]{2}{*}{$0440-003$} & 50 & 0.025 & 0.025 & $2.0 \mathrm{e} 4$ & $2.0 \mathrm{e} 3$ & 3.0 & 0.680 & 17 \\
\hline & 70 & 0.01 & - & $8.0 \mathrm{e} 4$ & 2.0 & 3.0 & 0.106 & 20 \\
\hline \multirow[t]{2}{*}{$0446+112$} & 50 & 0.08 & 1 & $4.0 \mathrm{e} 3$ & $1.5 \mathrm{e} 2$ & 2.0 & 0.810 & 20 \\
\hline & 90 & 0.08 & - & $1.0 \mathrm{e} 5$ & 1. & 3.5 & 0.011 & 20 \\
\hline \multirow[t]{2}{*}{$0454-463$} & 30 & 0.03 & 0.08 & $8.0 \mathrm{e} 3$ & $6.0 \mathrm{e} 2$ & 2.1 & 1.050 & 16 \\
\hline & 80 & $7 \mathrm{e}-3$ & - & $8.0 \mathrm{e} 4$ & $3.0 \mathrm{e} 4$ & 2.5 & 0.018 & 20 \\
\hline \multirow[t]{2}{*}{$0521-365$} & 50 & 1.00 & 0.10 & $5.0 \mathrm{e} 4$ & $2.0 \mathrm{e} 3$ & 2.5 & 3.141 & 1.4 \\
\hline & 50 & 0.60 & - & $7.0 \mathrm{e} 4$ & $3.5 \mathrm{e} 3$ & 2.7 & 2.580 & 1.6 \\
\hline \multirow[t]{2}{*}{$0528+134$} & 65 & 0.90 & 7 & $6.0 \mathrm{e} 3$ & $3.0 \mathrm{e} 2$ & 2.6 & 6.198 & 15 \\
\hline & 60 & 0.60 & - & $8.0 \mathrm{e} 4$ & 3. & 3.0 & 0.215 & 19 \\
\hline \multirow[t]{2}{*}{$0537-441$} & 70 & 0.04 & $1 \mathrm{e}-3$ & $7.0 \mathrm{e} 4$ & $4.0 \mathrm{e} 3$ & 2.2 & 0.325 & 15 \\
\hline & 50 & 0.05 & - & $1.0 \mathrm{e} 5$ & $4.0 \mathrm{e} 3$ & 2.5 & 0.430 & 15 \\
\hline \multirow{2}{*}{$0716+714$} & 30 & 0.02 & $7 \mathrm{e}-3$ & $3.0 \mathrm{e} 4$ & $2.0 \mathrm{e} 3$ & 2.6 & 1.813 & 11.5 \\
\hline & 50 & $3 e-3$ & - & $5.0 \mathrm{e} 4$ & 4.0 & 2.7 & 0.460 & 15 \\
\hline \multirow{2}{*}{$0735+178$} & 40 & 0.01 & $4 e-3$ & $3.0 \mathrm{e} 4$ & 3. & 3.0 & 0.833 & 14 \\
\hline & 50 & $3 e-3$ & - & $4 .($ & 5. & 3.3 & 0.408 & 17 \\
\hline \multirow[t]{2}{*}{$0804+499$} & 50 & 0.10 & 0.70 & $8.0 \mathrm{e} 3$ & $4.0 \mathrm{e} 2$ & 3.1 & 3.333 & 15 \\
\hline & 70 & 0.06 & - & $8.0 \mathrm{e} 4$ & $1.0 \mathrm{e} 4$ & 3.4 & 0.073 & 17 \\
\hline \multirow[t]{2}{*}{$0805-077$} & 40 & 0.20 & 2 & $6.0 \mathrm{e} 3$ & $4.0 \mathrm{e} 2$ & 2.5 & 2.483 & 17 \\
\hline & 90 & 0.09 & - & $1.0 \mathrm{e} 5$ & $9.0 \mathrm{e} 3$ & 3.7 & 0.037 & 20 \\
\hline \multirow[t]{2}{*}{$0827+234$} & 50 & 0.20 & 0.90 & $8.0 \mathrm{e} 3$ & $3.0 \mathrm{e} 2$ & 2.3 & 4.711 & 16 \\
\hline & 70 & 0.20 & - & $8.0 \mathrm{e} 4$ & $5.0 \mathrm{e} 3$ & 2.7 & 0.563 & 16 \\
\hline $0836+710$ & 50 & 0.70 & 6.00 & $7.0 \mathrm{e} 3$ & $1.8 \mathrm{e} 2$ & 3.1 & 8.814 & 18 \\
\hline & 70 & 0.30 & - & $5.0 \mathrm{e} 4$ & $4.0 \mathrm{e} 3$ & 3.0 & 0.282 & 17 \\
\hline $0917+449$ & 30 & 0.50 & 8 & $6.0 \mathrm{e} 3$ & $2.0 \mathrm{e} 2$ & 2.1 & 2.267 & 13 \\
\hline & 90 & 0.03 & - & $1.0 \mathrm{e} 5$ & $4.0 \mathrm{e} 3$ & 2.3 & 0.025 & 23 \\
\hline $0954+556$ & 50 & 0.01 & 0.02 & $1.5 \mathrm{e} 4$ & $5.0 \mathrm{e} 3$ & 1.7 & 0.527 & 15 \\
\hline & 70 & $4 e-3$ & - & $7.0 \mathrm{e} 4$ & $1.0 \mathrm{e} 4$ & 0.0 & 0.056 & 17 \\
\hline $0954+658$ & 30 & $4.5 \mathrm{e}-3$ & 0.025 & $6.0 \mathrm{e} 3$ & $9.0 \mathrm{e} 2$ & 3.6 & 2.040 & 13 \\
\hline & 60 & $7 e-4$ & - & $7.0 \mathrm{e} 4$ & $2.0 \mathrm{e} 4$ & 2.0 & 0.025 & 18 \\
\hline $1101+384$ & 5 & $6 e-3$ & $1 \mathrm{e}-3$ & $8.0 \mathrm{e} 5$ & $6.0 \mathrm{e} 4$ & 2.0 & 0.222 & 11 \\
\hline & 10 & $2 \mathrm{e}-3$ & - & $8.0 \mathrm{e} 5$ & $2.0 \mathrm{e} 5$ & 1.2 & 0.093 & 12 \\
\hline $1127-145$ & 60 & 0.15 & 0.80 & $8.0 \mathrm{e} 3$ & $3.0 \mathrm{e} 2$ & 2.3 & 1.862 & 15.5 \\
\hline & 70 & 0.10 & - & $8.0 \mathrm{e} 4$ & $1.5 \mathrm{e} 4$ & 2.3 & 0.048 & 17 \\
\hline $1156+295$ & 20 & 0.15 & 0.08 & $1.0 \mathrm{e} 4$ & $3.0 \mathrm{e} 3$ & 2.4 & 1.360 & 15 \\
\hline & 70 & 0.02 & - & $8.0 \mathrm{e} 4$ & $9.0 \mathrm{e} 3$ & 2.0 & 0.252 & 18 \\
\hline $1219+285$ & 20 & 1.e-3 & 4.e-3 & $1.0 \mathrm{e} 5$ & $5.0 \mathrm{e} 3$ & 4.2 & 1.178 & 11 \\
\hline & 70 & $5 . e-5$ & - & $7.0 \mathrm{e} 4$ & $4.0 \mathrm{e} 4$ & 0.0 & 0.036 & 20 \\
\hline $1222+216$ & 10 & 0.10 & 0.25 & $8.0 \mathrm{e} 3$ & $3.5 \mathrm{e} 2$ & 2.9 & 3.333 & 11 \\
\hline & 40 & 0.02 & - & $4.0 \mathrm{e} 4$ & $9.0 \mathrm{e} 3$ & 3.2 & 0.068 & 11 \\
\hline $1226+023$ & 10 & 1.00 & 1.50 & $1.0 \mathrm{e} 4$ & $8.0 \mathrm{e} 1$ & 3.2 & 8.900 & 6.5 \\
\hline & 40 & 0.06 & - & $3.0 \mathrm{e} 4$ & $3.5 \mathrm{e} 3$ & 3.2 & 0.456 & 7.0 \\
\hline
\end{tabular}


Table 2. continue

\begin{tabular}{|c|c|c|c|c|c|c|c|c|}
\hline $\begin{array}{l}\text { Source } \\
\text { (1) }\end{array}$ & $\begin{array}{c}R / 10^{15} \\
\quad(2)\end{array}$ & $\begin{array}{l}\ell_{\text {inj }} \\
(3)\end{array}$ & $\begin{array}{l}\ell_{\text {ext }} \\
(4)\end{array}$ & $\begin{array}{c}\gamma_{\max } \\
(5)\end{array}$ & $\begin{array}{c}\gamma_{\text {peak }} \\
(6)\end{array}$ & $\begin{array}{c}s \\
(7)\end{array}$ & $\begin{array}{c}B \\
(8)\end{array}$ & $\begin{array}{c}\delta \\
(9)\end{array}$ \\
\hline \multirow[t]{2}{*}{$1229-021$} & 40 & 0.08 & 1.00 & $8.0 \mathrm{e} 3$ & $3.0 \mathrm{e} 2$ & 3.8 & 2.356 & 12 \\
\hline & 50 & 0.03 & - & $2.0 \mathrm{e} 4$ & $1.0 \mathrm{e} 4$ & 4.0 & 0.105 & 16 \\
\hline \multirow[t]{2}{*}{$1253-055$} & 30 & 0.04 & 0.07 & 7.0e 3 & $8.0 \mathrm{e} 2$ & 3.2 & 1.360 & 18 \\
\hline & 80 & 0.04 & - & $2.0 \mathrm{e} 4$ & $2.0 \mathrm{e} 4$ & - & 0.059 & 15 \\
\hline \multirow[t]{2}{*}{$1313-333$} & 40 & 0.03 & 0.70 & $9.0 \mathrm{e} 3$ & $1.0 \mathrm{e} 3$ & 1.9 & 1.180 & 17 \\
\hline & 70 & 0.01 & - & $1.0 \mathrm{e} 5$ & $3.0 \mathrm{e} 4$ & 1.0 & 0.009 & 20 \\
\hline \multirow[t]{2}{*}{$1406-076$} & 60 & 0.05 & 0.10 & $1.0 \mathrm{e} 4$ & $3.0 \mathrm{e} 3$ & 1.5 & 1.080 & 21 \\
\hline & 70 & 0.08 & - & $6.0 \mathrm{e} 4$ & $1.5 \mathrm{e} 4$ & 2.3 & 0.206 & 18 \\
\hline \multirow[t]{2}{*}{$1424-418$} & 30 & 0.50 & 1.00 & $6.0 \mathrm{e} 3$ & $2.0 \mathrm{e} 2$ & 2.7 & 4.060 & 15 \\
\hline & 90 & 0.07 & - & $1.0 \mathrm{e} 5$ & $5.0 \mathrm{e} 3$ & 3.0 & 0.093 & 20 \\
\hline \multirow[t]{2}{*}{$1510-089$} & 20 & 0.05 & 0.80 & $1.0 \mathrm{e} 4$ & $1.2 \mathrm{e} 2$ & 3.3 & 5.890 & 13 \\
\hline & 30 & $4 e-3$ & - & $3.0 \mathrm{e} 4$ & $4.0 \mathrm{e} 3$ & 2.2 & 0.061 & 18 \\
\hline \multirow[t]{2}{*}{$1604+159$} & 20 & 0.01 & 0.10 & $1.0 \mathrm{e} 4$ & $9.0 \mathrm{e} 2$ & 2.1 & 0.960 & 15 \\
\hline & 50 & $2 \mathrm{e}-3$ & - & $7.0 \mathrm{e} 4$ & $3.0 \mathrm{e} 4$ & 2.1 & 0.011 & 18 \\
\hline \multirow[t]{2}{*}{$1606+106$} & 30 & 0.30 & 3.00 & $5.0 \mathrm{e} 3$ & $1.5 \mathrm{e} 2$ & 3.0 & 3.330 & 15 \\
\hline & 70 & 0.06 & - & $3.0 \mathrm{e} 4$ & $1.0 \mathrm{e} 4$ & 3.0 & 0.028 & 18 \\
\hline \multirow[t]{2}{*}{$1611+343$} & 30 & 0.20 & 1.00 & $5.0 \mathrm{e} 3$ & $2.0 \mathrm{e} 2$ & 2.6 & 3.041 & 16 \\
\hline & 50 & 0.20 & - & $6.0 \mathrm{e} 4$ & $1.3 \mathrm{e} 4$ & 3.3 & 0.105 & 13.5 \\
\hline \multirow[t]{2}{*}{$1622-253$} & 30 & 0.03 & 0.30 & $6.0 \mathrm{e} 3$ & $3.0 \mathrm{e} 2$ & 2.5 & 1.670 & 15 \\
\hline & 70 & 0.01 & - & $8.0 \mathrm{e} 4$ & $2.0 \mathrm{e} 4$ & 2.3 & 0.013 & 16 \\
\hline \multirow[t]{2}{*}{$1622-297$} & 20 & 0.10 & 1.00 & $2.5 \mathrm{e} 3$ & $6.0 \mathrm{e} 2$ & 1.5 & 2.150 & 23 \\
\hline & 80 & 0.03 & - & $5.0 \mathrm{e} 4$ & $3.0 \mathrm{e} 4$ & 2.1 & 0.007 & 21 \\
\hline \multirow[t]{2}{*}{$1633+382$} & 60 & 0.18 & 0.60 & $6.0 \mathrm{e} 3$ & $1.5 \mathrm{e} 3$ & 1.5 & 1.667 & 21 \\
\hline & 80 & 0.20 & - & $7.0 \mathrm{e} 4$ & $2.0 \mathrm{e} 4$ & 2.0 & 0.052 & 19 \\
\hline \multirow[t]{2}{*}{$1652+398$} & 5 & $2 \mathrm{e}-3$ & $5 e-4$ & $8.0 \mathrm{e} 5$ & $1.0 \mathrm{e} 4$ & 2.8 & 1.110 & 10 \\
\hline & 10 & $1 \mathrm{e}-3$ & - & $8.0 \mathrm{e} 5$ & $2.0 \mathrm{e} 4$ & 3.0 & 0.497 & 10 \\
\hline \multirow[t]{2}{*}{$1730-130$} & 30 & 0.04 & 0.40 & $3.0 \mathrm{e} 4$ & $4.0 \mathrm{e} 2$ & 2.8 & 5.440 & 17 \\
\hline & 60 & 0.02 & - & $6.0 \mathrm{e} 4$ & $6.0 \mathrm{e} 3$ & 2.4 & 0.192 & 16 \\
\hline \multirow[t]{2}{*}{$1739+522$} & 60 & 0.03 & 0.08 & $1.0 \mathrm{e} 4$ & $4.0 \mathrm{e} 2$ & 2.2 & 0.450 & 20 \\
\hline & 70 & 0.04 & - & $1.0 \mathrm{e} 5$ & $2.0 \mathrm{e} 4$ & 2.1 & 0.019 & 19 \\
\hline \multirow[t]{2}{*}{$1741-038$} & 60 & 0.05 & 0.90 & $8.0 \mathrm{e} 3$ & $2.5 \mathrm{e} 2$ & 3.8 & 2.150 & 17 \\
\hline & 60 & 0.05 & - & $2.0 \mathrm{e} 4$ & $1.0 \mathrm{e} 4$ & 3.0 & 0.048 & 17.5 \\
\hline \multirow[t]{2}{*}{$1933-400$} & 20 & 0.07 & 1.00 & $6.0 \mathrm{e} 3$ & $3.0 \mathrm{e} 2$ & 2.6 & 4.410 & 14 \\
\hline & 50 & 0.03 & - & $5.0 \mathrm{e} 4$ & $8.0 \mathrm{e} 3$ & 2.9 & 0.060 & 14 \\
\hline \multirow[t]{2}{*}{$2032+107$} & 20 & 0.06 & 0.20 & $6.0 \mathrm{e} 3$ & $3.0 \mathrm{e} 2$ & 3.0 & 3.850 & 12 \\
\hline & 50 & $5 \mathrm{e}-3$ & - & $5.0 \mathrm{e} 4$ & $4.0 \mathrm{e} 3$ & 3.3 & 0.118 & 20 \\
\hline \multirow[t]{2}{*}{$2052-474$} & 50 & 0.20 & 2.00 & $7.0 \mathrm{e} 3$ & $2.0 \mathrm{e} 2$ & 2.8 & 1.920 & 15 \\
\hline & 70 & 0.10 & - & $5.0 \mathrm{e} 4$ & $8.0 \mathrm{e} 3$ & 2.9 & 0.073 & 16 \\
\hline \multirow[t]{2}{*}{$2155-304$} & 20 & $2 \mathrm{e}-3$ & $3 e-4$ & $4.0 \mathrm{e} 5$ & $8.0 \mathrm{e} 3$ & 2.4 & 1.050 & 17 \\
\hline & 20 & $2 \mathrm{e}-3$ & - & $1.0 \mathrm{e} 6$ & $7.0 \mathrm{e} 3$ & 2.6 & 1.216 & 18 \\
\hline $2200+420$ & 8 & $8 \mathrm{e}-3$ & $3 e-4$ & $3.0 \mathrm{e} 5$ & $2.7 \mathrm{e} 3$ & 2.8 & 1.670 & 10 \\
\hline & 20 & $2 \mathrm{e}-3$ & - & $3.0 \mathrm{e} 5$ & $5.0 \mathrm{e} 3$ & 2.8 & 0.430 & 11 \\
\hline $2230+114$ & 40 & 0.80 & 5.00 & $1.0 \mathrm{e} 4$ & $1.0 \mathrm{e} 2$ & 3.1 & 8.600 & 10 \\
\hline & 70 & 0.03 & - & $3.0 \mathrm{e} 4$ & $6.0 \mathrm{e} 3$ & 2.9 & 0.077 & 18 \\
\hline $2251+158$ & 40 & 0.80 & 5.00 & $6.0 \mathrm{e} 3$ & $1.0 \mathrm{e} 2$ & 2.3 & 7.450 & 10 \\
\hline & 70 & 0.04 & - & $6.0 \mathrm{e} 4$ & $4.0 \mathrm{e} 3$ & 2.2 & 0.073 & 18 \\
\hline $2344+512$ & 8 & $1.4 \mathrm{e}-4$ & $1 \mathrm{e}-4$ & $7.0 \mathrm{e} 5$ & $4.0 \mathrm{e} 4$ & 3.7 & 0.470 & 14 \\
\hline & 10 & $2 \mathrm{e}-4$ & - & $8.0 \mathrm{e} 5$ & $4.5 \mathrm{e} 4$ & 3.5 & 0.220 & 13 \\
\hline
\end{tabular}


Table 3. Linear correlations for the EC model. (1), (2), (4), (5) parameters of the correlation of the form $y=m x+q$; (3) number of objects; (6) correlation coefficient; (7) probability of a random distribution; (8) sources considered.

\begin{tabular}{|c|c|c|c|c|c|c|c|}
\hline $\begin{array}{l}y \\
(1)\end{array}$ & $\begin{array}{l}x \\
(2)\end{array}$ & $\begin{array}{l}N \\
(3)\end{array}$ & $\begin{array}{r}m \\
(4)\end{array}$ & $\begin{array}{c}q \\
(5)\end{array}$ & $\begin{array}{c}r \\
(6)\end{array}$ & $\begin{array}{c}P \\
(7)\end{array}$ & $\begin{array}{l}\text { Objects } \\
(8)\end{array}$ \\
\hline \multirow[t]{3}{*}{$\log \gamma_{\text {peak }}$} & \multirow[t]{3}{*}{$\log \left(U_{\mathrm{r}}+U_{\mathrm{B}}\right)$} & 51 & $-0.63 \pm 0.04$ & $2.97 \pm 0.04$ & 0.902 & $7.2 \times 10^{-10}$ & All \\
\hline & & 37 & $-0.50 \pm 0.06$ & $2.88 \pm 0.05$ & 0.812 & $2.5 \times 10^{-9}$ & Only FSRQ \\
\hline & & 14 & $-0.80 \pm 0.12$ & $2.88 \pm 0.14$ & 0.886 & $2.4 \times 10^{-5}$ & Only BL Lacs \\
\hline \multirow[t]{3}{*}{$\log \gamma_{\text {peak }}$} & \multirow[t]{3}{*}{$\log U_{\mathrm{B}}$} & 51 & $-0.64 \pm 0.09$ & $2.33 \pm 0.10$ & 0.735 & $2.3 \times 10^{-9}$ & All \\
\hline & & 37 & $-0.43 \pm 0.08$ & $2.33 \pm 0.08$ & 0.676 & $4.5 \times 10^{-6}$ & Only FSRQ \\
\hline & & 14 & $-0.67 \pm 0.19$ & $2.70 \pm 0.28$ & 0.719 & $3.7 \times 10^{-3}$ & Only BL Lacs \\
\hline \multirow[t]{3}{*}{$\log \gamma_{\text {peak }}$} & \multirow[t]{3}{*}{$\log U_{\mathrm{r}}$} & 51 & $-0.56 \pm 0.04$ & $2.89 \pm 0.04$ & 0.911 & $1.2 \times 10^{-10}$ & All \\
\hline & & 37 & $-0.50 \pm 0.06$ & $2.85 \pm 0.05$ & 0.818 & $7.9 \times 10^{-11}$ & Only FSRQ \\
\hline & & 14 & $-0.64 \pm 0.10$ & $2.82 \pm 0.15$ & 0.875 & $4.0 \times 10^{-5}$ & Only BL Lacs \\
\hline \multirow[t]{3}{*}{$\log \gamma_{\text {peak }}$} & \multirow[t]{3}{*}{$\log \ell_{\text {ext }}$} & 51 & $-0.48 \pm 0.04$ & $2.47 \pm 0.05$ & 0.890 & $7.1 \times 10^{-10}$ & All \\
\hline & & 37 & $-0.54 \pm 0.07$ & $2.47 \pm 0.05$ & 0.799 & $1.6 \times 10^{-9}$ & Only FSRQ \\
\hline & & 14 & $-0.49 \pm 0.11$ & $2.39 \pm 0.29$ & 0.794 & $7.0 \times 10^{-4}$ & Only BL Lacs \\
\hline \multirow[t]{3}{*}{$\log \gamma_{\text {peak }}$} & \multirow[t]{3}{*}{$\log \ell_{\text {inj }}$} & 51 & $-0.61 \pm 0.08$ & $2.11 \pm 0.12$ & 0.735 & $3.4 \times 10^{-9}$ & All \\
\hline & & 37 & $-0.41 \pm 0.12$ & $2.23 \pm 0.14$ & 0.491 & $2.0 \times 10^{-3}$ & Only FSRQ \\
\hline & & 14 & $-0.48 \pm 0.21$ & $2.59 \pm 0.48$ & 0.566 & $3.5 \times 10^{-2}$ & Only BL Lacs \\
\hline \multirow[t]{3}{*}{$\log \gamma_{\text {peak }}$} & \multirow{3}{*}{$\log \left(\ell_{\mathrm{inj}}+\ell_{\text {ext }}\right)$} & 51 & $-0.58 \pm 0.05$ & $2.54 \pm 0.06$ & 0.871 & $1.7 \times 10^{-10}$ & All \\
\hline & & 37 & $-0.55 \pm 0.09$ & $2.54 \pm 0.05$ & 0.740 & $1.7 \times 10^{-7}$ & Only FSRQ \\
\hline & & 14 & $-0.61 \pm 0.14$ & $2.49 \pm 0.29$ & 0.776 & $1.1 \times 10^{-3}$ & Only BL Lacs \\
\hline \multirow[t]{3}{*}{$\log \gamma_{\text {peak }}$} & \multirow[t]{3}{*}{$\log L_{\mathrm{C}} / L_{\mathrm{syn}}$} & 51 & $-0.70 \pm 0.11$ & $3.53 \pm 0.12$ & 0.690 & $2.2 \times 10^{-8}$ & All \\
\hline & & 37 & $-0.44 \pm 0.18$ & $3.15 \pm 0.23$ & 0.381 & $2.0 \times 10^{-2}$ & Only FSRQ \\
\hline & & 14 & $-0.46 \pm 0.28$ & $3.67 \pm 0.16$ & 0.427 & $1.3 \times 10^{-1}$ & Only BL Lacs \\
\hline \multirow[t]{3}{*}{$\log L_{\mathrm{C}} / L_{\mathrm{syn}}$} & \multirow[t]{3}{*}{$\log \ell_{\text {inj }}$} & 51 & $0.49 \pm 0.09$ & $1.54 \pm 0.14$ & 0.601 & $2.3 \times 10^{-6}$ & All \\
\hline & & 37 & $0.02 \pm 0.12$ & $1.18 \pm 0.13$ & 0.027 & $8.7 \times 10^{-1}$ & Only FSRQ \\
\hline & & 14 & $0.47 \pm 0.18$ & $1.15 \pm 0.41$ & 0.599 & $2.4 \times 10^{-2}$ & Only BL Lacs \\
\hline \multirow[t]{3}{*}{$\log L_{\mathrm{C}} / L_{\mathrm{syn}}$} & \multirow[t]{3}{*}{$\log \ell_{\text {ext }}$} & 51 & $0.42 \pm 0.05$ & $1.28 \pm 0.07$ & 0.797 & $2.2 \times 10^{-9}$ & All \\
\hline & & 37 & $0.25 \pm 0.09$ & $1.27 \pm 0.07$ & 0.426 & $8.6 \times 10^{-3}$ & Only FSRQ \\
\hline & & 14 & $0.38 \pm 0.13$ & $1.07 \pm 0.34$ & 0.649 & $1.2 \times 10^{-2}$ & Only BL Lacs \\
\hline \multirow[t]{3}{*}{$\log L_{\mathrm{C}} / L_{\mathrm{syn}}$} & \multirow[t]{3}{*}{$\log \left(\ell_{\mathrm{inj}}+\ell_{\text {ext }}\right)$} & 51 & $0.50 \pm 0.06$ & $1.22 \pm 0.07$ & 0.771 & $6.7 \times 10^{-10}$ & All \\
\hline & & 37 & $0.20 \pm 0.10$ & $1.23 \pm 0.07$ & 0.311 & $6.1 \times 10^{-2}$ & Only FSRQ \\
\hline & & 14 & $0.55 \pm 0.14$ & $1.15 \pm 0.28$ & 0.749 & $2.0 \times 10^{-3}$ & Only BL Lacs \\
\hline \multirow{3}{*}{$\log L_{\mathrm{C}} / L_{\mathrm{syn}}$} & \multirow{3}{*}{$\log L_{\mathrm{inj}}^{\mathrm{obs}}$} & 51 & $0.40 \pm 0.06$ & $-18.44 \pm 2.81$ & 0.702 & $8.7 \times 10^{-9}$ & All \\
\hline & & 37 & $0.17 \pm 0.08$ & $-7.28 \pm 4.03$ & 0.335 & $4.2 \times 10^{-2}$ & Only FSRQ \\
\hline & & 14 & $0.29 \pm 0.12$ & $-13.44 \pm 5.61$ & 0.573 & $3.2 \times 10^{-2}$ & Only BL Lacs \\
\hline $\log L_{\mathrm{C}} / L_{\mathrm{syn}}$ & $\log \nu_{\text {peak }}^{\text {obs }}$ & 51 & $-0.45 \pm 0.06$ & $11.57 \pm 1.40$ & 0.736 & $1.4 \times 10^{-9}$ & All \\
\hline & & 37 & $-0.25 \pm 0.08$ & $6.92 \pm 1.93$ & 0.449 & $5.3 \times 10^{-3}$ & Only FSRQ \\
\hline & & 14 & $-0.31 \pm 0.14$ & $7.88 \pm 3.45$ & 0.544 & $4.4 \times 10^{-2}$ & Only BL Lacs \\
\hline $\log L_{\mathrm{C}} / L_{\mathrm{syn}}$ & $\log B$ & 51 & $0.37 \pm 0.24$ & $0.81 \pm 0.11$ & 0.217 & $1.3 \times 10^{-1}$ & All \\
\hline & & 37 & $-0.15 \pm 0.19$ & $1.26 \pm 0.09$ & 0.132 & $4.3 \times 10^{-1}$ & Only FSRQ \\
\hline & & 14 & $-0.25 \pm 0.50$ & $0.14 \pm 0.16$ & 0.145 & $6.2 \times 10^{-1}$ & Only BL Lacs \\
\hline $\log \ell_{\text {ext }}$ & $\log \ell_{\text {inj }}$ & 51 & $1.28 \pm 0.13$ & $0.76 \pm 0.19$ & 0.822 & $6.3 \times 10^{-10}$ & All \\
\hline & & 37 & $0.83 \pm 0.16$ & $0.52 \pm 0.17$ & 0.671 & $5.5 \times 10^{-6}$ & Only FSRQ \\
\hline & & 14 & $0.82 \pm 0.31$ & $-0.71 \pm 0.71$ & 0.605 & $2.2 \times 10^{-2}$ & Only BL Lacs \\
\hline $\log U_{B}$ & $\log U_{r}$ & 51 & $0.57 \pm 0.06$ & $-0.86 \pm 0.06$ & 0.812 & $1.2 \times 10^{-10}$ & All \\
\hline & & 37 & $0.82 \pm 0.08$ & $-1.05 \pm 0.07$ & 0.857 & $5.7 \times 10^{-10}$ & Only FSRQ \\
\hline & & 14 & $0.54 \pm 0.16$ & $-0.69 \pm 0.24$ & 0.693 & $6.0 \times 10^{-3}$ & Only BL Lacs \\
\hline
\end{tabular}


Table 4. Linear correlations for the SSC model. (1), (2), (4), (5) parameters of the correlation of the form $y=m x+q$; (3) number of objects; (6) correlation coefficient; (7) probability of a random distribution; (8) sources considered.

\begin{tabular}{|c|c|c|c|c|c|c|c|}
\hline $\begin{array}{l}y \\
(1)\end{array}$ & $\begin{array}{l}x \\
(2)\end{array}$ & $\begin{array}{l}N \\
(3)\end{array}$ & $\begin{array}{r}m \\
(4)\end{array}$ & $\begin{array}{r}q \\
(5)\end{array}$ & $\begin{array}{c}r \\
(6)\end{array}$ & $\begin{array}{c}P \\
(7)\end{array}$ & $\begin{array}{l}\text { Objects } \\
(8)\end{array}$ \\
\hline \multirow[t]{3}{*}{$\log \gamma_{\text {peak }}$} & \multirow[t]{3}{*}{$\log \left(U_{\mathrm{r}}+U_{\mathrm{B}}\right)$} & 51 & $-0.29 \pm 0.05$ & $3.37 \pm 0.13$ & 0.616 & $1.5 \times 10^{-6}$ & All \\
\hline & & 37 & $-0.24 \pm 0.05$ & $3.43 \pm 0.12$ & 0.627 & $3.3 \times 10^{-5}$ & Only FSRQ \\
\hline & & 14 & $-0.36 \pm 0.13$ & $3.29 \pm 0.31$ & 0.638 & $1.4 \times 10^{-2}$ & Only BL Lacs \\
\hline \multirow[t]{3}{*}{$\log \gamma_{\text {peak }}$} & \multirow[t]{3}{*}{$\log U_{\mathrm{B}}$} & 51 & $-0.15 \pm 0.04$ & $3.51 \pm 0.15$ & 0.466 & $5.7 \times 10^{-4}$ & All \\
\hline & & 37 & $-0.17 \pm 0.04$ & $3.35 \pm 0.14$ & 0.627 & $3.2 \times 10^{-5}$ & Only FSRQ \\
\hline & & 14 & $-0.23 \pm 0.11$ & $3.48 \pm 0.32$ & 0.524 & $5.4 \times 10^{-2}$ & Only BL Lacs \\
\hline \multirow{3}{*}{$\log \gamma_{\text {peak }}$} & \multirow[t]{3}{*}{$\log U_{\mathrm{r}}$} & 51 & $-0.31 \pm 0.05$ & $3.29 \pm 0.13$ & 0.642 & $3.7 \times 10^{-7}$ & All \\
\hline & & 37 & $-0.25 \pm 0.05$ & $3.41 \pm 0.13$ & 0.627 & $3.3 \times 10^{-5}$ & Only FSRQ \\
\hline & & 14 & $-0.41 \pm 0.13$ & $3.07 \pm 0.35$ & 0.672 & $8.5 \times 10^{-3}$ & Only BL Lacs \\
\hline \multirow[t]{3}{*}{$\log \gamma_{\text {peak }}$} & \multirow[t]{3}{*}{$\log \ell_{i n j}$} & 51 & $-0.19 \pm 0.06$ & $3.70 \pm 0.11$ & 0.434 & $1.5 \times 10^{-3}$ & All \\
\hline & & 37 & $-0.19 \pm 0.08$ & $3.72 \pm 0.12$ & 0.365 & $2.6 \times 10^{-2}$ & Only FSRQ \\
\hline & & 14 & $-0.36 \pm 0.16$ & $3.16 \pm 0.45$ & 0.539 & $4.7 \times 10^{-2}$ & Only BL Lacs \\
\hline \multirow[t]{3}{*}{$\log \gamma_{\text {peak }}$} & \multirow[t]{3}{*}{$\log L_{\mathrm{C}} / L_{\mathrm{syn}}$} & 51 & $0.05 \pm 0.07$ & $3.96 \pm 0.10$ & 0.100 & $4.8 \times 10^{-1}$ & All \\
\hline & & 37 & $0.32 \pm 0.09$ & $3.52 \pm 0.14$ & 0.504 & $1.5 \times 10^{-3}$ & Only FSRQ \\
\hline & & 14 & $0.12 \pm 0.23$ & $4.09 \pm 0.16$ & 0.143 & $6.2 \times 10^{-1}$ & Only BL Lacs \\
\hline \multirow[t]{3}{*}{$\log L_{\mathrm{C}} / L_{\mathrm{syn}}$} & \multirow[t]{3}{*}{$\log \ell_{i n j}$} & 51 & $0.47 \pm 0.10$ & $1.94 \pm 0.19$ & 0.555 & $2.4 \times 10^{-5}$ & All \\
\hline & & 37 & $-0.03 \pm 0.14$ & $1.41 \pm 0.20$ & 0.031 & $8.6 \times 10^{-1}$ & Only FSRQ \\
\hline & & 14 & $0.24 \pm 0.23$ & $0.95 \pm 0.64$ & 0.292 & $3.1 \times 10^{-1}$ & Only BL Lacs \\
\hline \multirow[t]{3}{*}{$\log L_{\mathrm{C}} / L_{\mathrm{syn}}$} & \multirow[t]{3}{*}{$\log L_{\mathrm{inj}}^{\mathrm{obs}}$} & 51 & $0.45 \pm 0.06$ & $-20.52 \pm 2.94$ & 0.725 & $5.9 \times 10^{-9}$ & All \\
\hline & & 37 & $0.27 \pm 0.08$ & $-11.84 \pm 3.90$ & 0.499 & $1.7 \times 10^{-3}$ & Only FSRQ \\
\hline & & 14 & $0.28 \pm 0.15$ & $-13.00 \pm 6.88$ & 0.487 & $7.7 \times 10^{-2}$ & Only BL Lacs \\
\hline \multirow[t]{3}{*}{$\log L_{\mathrm{C}} / L_{\mathrm{syn}}$} & \multirow{3}{*}{$\log \nu_{\text {peak }}^{\text {obs }}$} & 51 & $-0.54 \pm 0.14$ & $14.57 \pm 3.39$ & 0.493 & $2.4 \times 10^{-4}$ & All \\
\hline & & 37 & $-0.15 \pm 0.16$ & $5.19 \pm 4.09$ & 0.156 & $3.5 \times 10^{-1}$ & Only FSRQ \\
\hline & & 14 & $-0.25 \pm 0.21$ & $6.62 \pm 5.35$ & 0.324 & $2.6 \times 10^{-1}$ & Only BL Lacs \\
\hline \multirow[t]{3}{*}{$\log L_{\mathrm{C}} / L_{\mathrm{syn}}$} & \multirow[t]{3}{*}{$\log B$} & 51 & $-0.98 \pm 0.11$ & $0.10 \pm 0.13$ & 0.783 & $1.2 \times 10^{-9}$ & All \\
\hline & & 37 & $-0.74 \pm 0.07$ & $0.58 \pm 0.09$ & 0.864 & $1.5 \times 10^{-11}$ & Only FSRQ \\
\hline & & 14 & $-0.82 \pm 0.20$ & $-0.30 \pm 0.19$ & 0.761 & $1.6 \times 10^{-3}$ & Only BL Lacs \\
\hline \multirow[t]{3}{*}{$\log U_{B}$} & \multirow[t]{3}{*}{$\log U_{r}$} & 51 & $1.21 \pm 0.13$ & $-0.64 \pm 0.33$ & 0.791 & $2.3 \times 10^{-9}$ & All \\
\hline & & 37 & $1.39 \pm 0.08$ & $-0.57 \pm 0.19$ & 0.945 & $6.3 \times 10^{-11}$ & Only FSRQ \\
\hline & & 14 & $1.22 \pm 0.21$ & $0.27 \pm 0.57$ & 0.857 & $9.0 \times 10^{-5}$ & Only BL Lacs \\
\hline
\end{tabular}

Table 5. The input parameters for the EC model reproducing the average SEDs determined by Fossati et al. (1998) by dividing into radio luminosity $\left(\mathrm{L}_{\mathrm{R}}\right)$ bins BL Lacs and FSRQ belonging to complete samples. (1) Radio luminosity bin; (2) region size in units of $10^{15}$ cm; (3), (4) compactnesses in injected particles and external radiation field; (5) maximum energy of the injected particles; (6) energy of the peak of the stationary electron distribution; (7) spectral index of the injected particles; (8) magnetic field intensity (in Gauss). In the fits the relativistic Doppler factor (column 9) has been fixed at the value $\delta=15$.

\begin{tabular}{lcccccccc}
\hline $\log \mathrm{L}_{\mathrm{R}}$ & $R / 10^{15}$ & $\ell_{\text {inj }}$ & $\ell_{\text {ext }}$ & $\gamma_{\max }$ & $\gamma_{\text {peak }}$ & $s$ & $B$ & $\delta$ \\
$(1)$ & $(2)$ & $(3)$ & $(4)$ & $(5)$ & $(6)$ & $(7)$ & $(8)$ & $(9)$ \\
\hline 41.5 & 10 & $7 \mathrm{e}-4$ & $1 \mathrm{e}-5$ & $1.0 \mathrm{e} 6$ & $2.5 \mathrm{e} 4$ & 3.0 & 0.441 & 15 \\
42.5 & 10 & $1 \mathrm{e}-3$ & $1 \mathrm{e}-3$ & $2.0 \mathrm{e} 5$ & $2.0 \mathrm{e} 3$ & 2.8 & 0.745 & 15 \\
43.5 & 30 & $5 \mathrm{e}-3$ & $2 \mathrm{e}-2$ & $3.0 \mathrm{e} 4$ & $5.0 \mathrm{e} 2$ & 3.0 & 1.075 & 15 \\
44.5 & 40 & $5 \mathrm{e}-2$ & 0.1 & $1.5 \mathrm{e} 4$ & $3.5 \mathrm{e} 2$ & 2.5 & 1.178 & 15 \\
45.5 & 50 & $5 \mathrm{e}-1$ & 5.0 & $6.0 \mathrm{e} 3$ & $1.5 \mathrm{e} 2$ & 2.8 & 6.082 & 15 \\
\hline
\end{tabular}


Table 6. Results of the principal component analysis. EV stands for eigenvector. The first row of the table lists the percentage of the correlation accounted for by the different eigenvectors.

\begin{tabular}{lrrrrrr}
\hline Variable & $\mathrm{EV}_{1}$ & $\mathrm{EV}_{2}$ & $\mathrm{EV}_{3}$ & $\mathrm{EV}_{4}$ & $\mathrm{EV}_{5}$ & $\mathrm{EV}_{6}$ \\
\hline$\%$ & 44.55 & 27.08 & 13.17 & 9.0 & 3.52 & 2.66 \\
\hline$\delta$ & -0.2408 & -0.5922 & -0.3525 & 0.5140 & 0.1872 & -0.4098 \\
$\ell_{\text {inj }}$ & 0.5608 & 0.0623 & -0.0525 & -0.3317 & 0.0915 & -0.7486 \\
$\ell_{\text {ext }}$ & 0.5242 & -0.1578 & -0.3110 & 0.2645 & -0.7031 & 0.1982 \\
$\gamma_{\text {peak }}$ & -0.2086 & 0.4119 & -0.8594 & -0.2063 & 0.0652 & 0.0376 \\
$U_{B}$ & 0.5510 & 0.0746 & -0.1082 & 0.3196 & 0.6654 & 0.3663 \\
$R$ & 0.0748 & -0.6673 & -0.1611 & -0.6412 & 0.1234 & 0.3111 \\
\hline
\end{tabular}

